\title{
Robust Position Control of PMSM Using Fractional-Order Sliding Mode Controller
}

\author{
Jiacai Huang, ${ }^{1}$ Hongsheng Li, ${ }^{1}$ \\ YangQuan Chen, ${ }^{2}$ and Qinghong $\mathrm{Xu}^{1}$ \\ ${ }^{1}$ Automation Department, Nanjing Institute of Technology, Nanjing 211167, China \\ ${ }^{2}$ School of Engineering, University of California, Merced, CA 95343, USA \\ Correspondence should be addressed to Jiacai Huang, zdhxhjc@njit.edu.cn
}

Received 29 June 2012; Revised 26 August 2012; Accepted 26 August 2012

Academic Editor: Dumitru Băleanu

Copyright (C) 2012 Jiacai Huang et al. This is an open access article distributed under the Creative Commons Attribution License, which permits unrestricted use, distribution, and reproduction in any medium, provided the original work is properly cited.

\begin{abstract}
A new robust fractional-order sliding mode controller (FOSMC) is proposed for the position control of a permanent magnet synchronous motor (PMSM). The sliding mode controller (SMC), which is insensitive to uncertainties and load disturbances, is studied widely in the application of PMSM drive. In the existing SMC method, the sliding surface is usually designed based on the integer-order integration or differentiation of the state variables, while in this proposed robust FOSMC algorithm, the sliding surface is designed based on the fractional-order calculus of the state variables. In fact, the conventional SMC method can be seen as a special case of the proposed FOSMC method. The performance and robustness of the proposed method are analyzed and tested for nonlinear load torque disturbances, and simulation results show that the proposed algorithm is more robust and effective than the conventional SMC method.
\end{abstract}

\section{Introduction}

Permanent magnet synchronous motor (PMSM) has many applications in industries due to its superior features such as compact structure, high efficiency, high torque to inertia ratio, and high power density [1]. To get fast four-quadrant operation, good acceleration, and smooth starting, the field-oriented control or vector control is used in the design of PMSM drives [1-4]. However, the PMSM is a typical high nonlinear, multivariable coupled system, and its performance is sensitive to external load disturbances, parameter changes in plant, and unmodeled and nonlinear dynamics. To achieve good dynamic response, some 
robust control strategies such as nonlinear control $[5,6]$, adaptive control $[7,8], H_{\infty}$ control [9-11], and sliding mode control (SMC) [12-18] have been developed.

The SMC is a powerful nonlinear control technique and has been widely used for speed and position control of PMSM system, because it provides a fast dynamic response and is insensitive to external load disturbances and parameter variations. In [14], a fuzzy sliding mode controller was proposed for the speed and position control of PMSM. In [15], a hybrid controller (HC) which consists of a parallel connected sliding mode controller and a neurofuzzy controller was proposed for the speed control of PMSM. In [16], a robust wavelet-neural-network sliding mode controller was proposed which can achieve favourable decoupling control and high-precision speed tracking performance of PMSM. The design of the SMC mainly contains two steps: the first step is to select the sliding surface, which is usually the linear manifold of the state variables and can guarantee the asymptotic stability; the second step is to determine the control output, which drives the system state to the designed sliding surface and constrains the state to the surface subsequently. Usually, the design of sliding surface for a PMSM is limited to integer order, which means that the sliding surface is constructed by the integer-order integration or differentiation of the state variables.

Fractional calculus has a 300-year-old history, and for a long time, it was considered as a pure theoretical subject with nearly no applications. In recent decades, not only the theory of fractional-order calculus is developed greatly, but also the application of fractional controller attracts increasing attention due to the higher degree of freedom provided [19-36]. In [33] the fractional-order adaptation law for integer-order sliding mode control is studied and applied in the 2DOF robot. In [34], the synchronization of chaotic and uncertain DuffingHolmes system has been done using the sliding mode control strategy and fractional order mathematics. In [35], a robust fractional-order proportion-plus-differential (FOPD) controller for the control of PMSM was proposed. In control practice, it is useful to consider the fractional-order controller design for an integer-order plant. This is due to the fact that the plant model may have already been obtained as an integer-order model in classical sense. In most cases, our objective of using fractional calculus is to apply the fractional-order control (FOC) to enhance the system control performance.

This paper applies the fractional calculus into the sliding surface design and proposes a robust fractional-order sliding mode controller (FOSMC) for the position control of a PMSM. The rest of this paper is organized as follows. In Section 2, the fractional-order calculus operation is introduced. In Section 3, the mathematical model of PMSM is given. In Section 4, the conventional integer-order SMC for PMSM is reviewed. In Section 5, the FOSMC method for position control of PMSM is derived. In Section 6, the robustness of the proposed FOSMC method is analyzed. In Section 7, the effectiveness of the proposed algorithm is illustrated through numerical examples and compared with the conventional integer-order SMC. In Section 8, the guidance for parameters selection and design is given. Finally, conclusions are presented in Section 9.

\section{Fractional Calculus}

Fractional calculus has been known since the development of the integer-order calculus, but for a long time, it has been considered as a sole mathematical problem. In recent decades, fractional calculus has become an interesting topic among system analyses and control fields. 
Fractional calculus is a generalization of integer-order integration and differentiation to non-integer-order ones. Let symbol ${ }_{a} D_{t}^{\lambda}$ denote the fractional-order fundamental operator, defined as follows $[20,21]$ :

$$
D^{\lambda} \triangleq{ }_{a} D_{t}^{\lambda}= \begin{cases}\frac{d^{\lambda}}{d t^{\lambda}} & R(\lambda)>0, \\ 1 & R(\lambda)=0 \\ \int_{a}^{t}(d \tau)^{-\lambda} & R(\lambda)<0,\end{cases}
$$

where $a$ and $t$ are the limits of the operation, $\lambda$ is the order of the operation, and generally $\lambda \in R$ and $\lambda$ can be a complex number.

The two most used definitions for the general fractional differentiation and integration are the Grunwald-Letnikov (GL) definition [22] and the Riemann-Liouville (RL) definition [23]. The GL is given by

$$
{ }_{a} D_{t}^{\lambda} f(t)=\lim _{h \rightarrow 0} h^{-\lambda} \sum_{j=0}^{[(t-a) / h]}(-1)^{j}\left(\begin{array}{l}
\lambda \\
j
\end{array}\right) f(t-j h),
$$

where $[\cdot]$ means the integer part.

The RL definition is given as

$$
{ }_{a} D_{t}^{\lambda} f(t)=\frac{1}{\Gamma(n-\lambda)} \frac{d^{n}}{d t^{n}} \int_{a}^{t} \frac{f(\tau)}{(t-\tau)^{\lambda-n+1}} d \tau
$$

where $n-1<\lambda<n$, and $\Gamma(\cdot)$ is the Gamma function.

Having zero initial conditions, the Laplace transformation of the RL definition for a fractional-order $\lambda$ is given by

$$
L\left\{{ }_{a} D_{t}^{\lambda} f(t)\right\}=s^{\lambda} F(s)
$$

where $F(s)$ is the Laplace transformation of $f(t)$.

Distinctly, the fractional-order operator has more degrees of freedom than that with integer order. It is likely that a better performance can be obtained with the proper choice of order.

\section{Mathematical Model of PMSM}

The PMSM is composed of a stator and a rotor; the rotor is made by a permanent magnet, and the stator has 3-phase windings which are distributed sinusoidally. To get the model of the PMSM, some assumptions are made: (a) the eddy current and hysteresis losses are ignored; (b) magnetic saturation is neglected; (c) no damp winding is on the rotor; (d) the induced EMF is 
sinusoidal. Under the above assumptions, the mathematics model of a PMSM can be described in the rotor rotating reference frame as follows [2]:

$$
\begin{gathered}
u_{d}=R i_{d}-\omega_{e} L_{q} i_{q}+L_{d} \frac{d i_{d}}{d t}, \\
u_{q}=R i_{q}+\omega_{e} L_{d} i_{d}+\omega_{e} \psi_{f}+L_{q} \frac{d i_{q}}{d t} .
\end{gathered}
$$

In the above equations, $u_{d}$ and $u_{q}$ are voltages in the $d$ - and $q$-axes, $i_{d}$ and $i_{q}$ are currents in the $d$ - and $q$-axes, $L_{d}$ and $L_{q}$ are inductances in the $d$ - and $q$-axes, $R$ is the stator resistance, $\omega_{e}$ is the electrical angular velocity, and $\psi_{f}$ is the flux linkage of the permanent magnet.

The corresponding electromagnetic torque is as follows:

$$
T_{e}=P\left[\psi_{f} i_{q}+\left(L_{d}-L_{q}\right) i_{d} i_{q}\right]
$$

where $T_{e}$ is the electromagnetic torque, and $P$ is the pole number of the rotor.

For surface PMSM, we have $L_{d}=L_{q}$; thus, the electromagnetic torque equation is rewritten as follows:

$$
T_{e}=P \psi_{f} i_{q}
$$

The associated mechanical equation is as follows:

$$
T_{e}-T_{L}=J \frac{d \omega_{m}}{d t}+B \omega_{m}
$$

where $J$ is the motor moment inertia constant, $T_{L}$ is the external load torque, $B$ is the viscous friction coefficient, and $\omega_{m}$ is the rotor angular speed, and it satisfies

$$
\omega_{e}=P \omega_{m}
$$

In this paper, the $i_{d}=0$ decoupled control method is applied, which means that there is no demagnetization effect, and the electromagnetic torque and the armature current are the linear relationship.

\section{Review of Conventional SMC}

\subsection{State Equations of PMSM System}

The object of the designed controller is to make the position $\theta_{m}$ strictly follow its desired signal $\theta_{\text {ref. Let }}$

$$
\begin{gathered}
x_{1}=\theta_{\text {ref }}-\theta_{m}, \\
x_{2}=\dot{x}_{1}=\dot{\theta}_{\text {ref }}-\dot{\theta}_{m},
\end{gathered}
$$


where $x_{1}$ and $x_{2}$ are the state error variables of the PMSM system,

$$
\begin{aligned}
& \dot{\theta}_{m}=\omega_{m}, \\
& \ddot{\theta}_{m}=\dot{\omega}_{m} .
\end{aligned}
$$

From (4.1) and (4.2), it is obvious that

$$
\begin{gathered}
\dot{x}_{1}=x_{2}=\dot{\theta}_{\text {ref }}-\dot{\theta}_{m}, \\
\dot{x}_{2}=\ddot{\theta}_{\text {ref }}-\ddot{\theta}_{m}=\ddot{\theta}_{\text {ref }}-\dot{\omega}_{m} .
\end{gathered}
$$

Substituting (3.3) and (3.4) into (4.3), we have

$$
\dot{x}_{2}=\ddot{\theta}_{\mathrm{ref}}-\frac{1}{J}\left[P \psi_{f} i_{q}-T_{L}-B \omega_{m}\right] .
$$

Then the state-space equation of the PMSM control system can be written as follows:

$$
\left[\begin{array}{l}
\dot{x}_{1} \\
\dot{x}_{2}
\end{array}\right]=\left[\begin{array}{ll}
0 & 1 \\
0 & 0
\end{array}\right]\left[\begin{array}{l}
x_{1} \\
x_{2}
\end{array}\right]+\left[\begin{array}{l}
0 \\
E
\end{array}\right] U+\left[\begin{array}{l}
0 \\
F
\end{array}\right]
$$

where

$$
E=-\frac{P \psi_{f}}{J}, \quad F=\ddot{\theta}_{\text {ref }}+\frac{T_{L}+B \omega_{m}}{J}, \quad U=i_{q}
$$

\subsection{The Conventional Integer-Order SMC}

The design of the SMC usually consists of two steps. Firstly, the sliding surface is designed such that the system motion on the sliding mode can satisfy the design specifications; secondly, a control law is designed to drive the system state to the designed sliding surface and constrains the state to the surface subsequently.

The conventional integer-order sliding surface $S$ is designed as follows [4]:

$$
S=c x_{1}+x_{2}
$$

where $c$ is set as a positive constant, and the derivative of (4.7) is as follows:

$$
\dot{S}=c \dot{x}_{1}+\dot{x}_{2}
$$

Substituting (4.3) and (4.4) into (4.8), we have

$$
\dot{S}=c x_{2}+\ddot{\theta}_{\text {ref }}-\frac{1}{J}\left[P \psi_{f} i_{q}-T_{L}-B \omega_{m}\right]
$$


When $T_{L}=0$, and forcing $\dot{S}=0$, then the control output is obtained as follows:

$$
U_{\mathrm{eq}}=i_{q}=\frac{J}{P \psi_{f}}\left(c x_{2}+\ddot{\theta}_{\mathrm{ref}}+\frac{1}{J} B \omega_{m}\right)
$$

Here, $U_{\text {eq }}$ is the equivalent control, which keeps the state variables on the sliding surface.

When the system has immeasurable disturbances with upper limit $T_{L \text {-max }}$, then the final control output can be given as

$$
U=i_{q}=U_{\mathrm{eq}}+k \operatorname{sgn}(S)=\frac{J}{P \psi_{f}}\left(c x_{2}+\ddot{\theta}_{\mathrm{ref}}+\frac{1}{J} B \omega_{m}\right)+k \operatorname{sgn}(S),
$$

where $k$ is a positive switch gain, and $\operatorname{sgn}(\cdot)$ denotes the sign function defined as

$$
\operatorname{sgn}(S)= \begin{cases}1 & S>0 \\ 0 & S=0 \\ -1 & S<0\end{cases}
$$

\subsection{Stability Analysis}

The Lyapunov function is defined as

$$
V=\frac{1}{2} S^{2}
$$

According to the Lyapunov stability theorem, the sliding surface reaching condition is $S \dot{S}<0$. Taking the derivative of (4.13) and substituting (4.11) into (4.9), we have

$$
\dot{V}=S \dot{S}=S\left[\frac{T_{L-\max }}{J}-\frac{P \psi_{f}}{J} k \operatorname{sgn}(S)\right]
$$

From (4.14), it is obvious that when

$$
k>\frac{T_{L-\max }}{P \psi_{f}}
$$

then $S \dot{S}<0$, and the system is globally and asymptotically stable; $S$ and $\dot{S}$ will approach zero in a finite time duration.

\section{Proposed Fractional-Order SMC (FOSMC)}

In this section, the fractional-order sliding mode controller (FOSMC) for the position control of PMSM will be proposed. 


\subsection{Design of Fractional-Order Sliding Surface}

First, the fractional-order sliding surface is designed as follows:

$$
S=k_{p} x_{1}+k_{d} D^{\mu} x_{1}=k_{p} x_{1}+k_{d} D^{\mu-1} x_{2}
$$

where $k_{p}$ and $k_{d}$ are set as positive constants, the function $D^{\mu}$ is defined as (2.1), and $0<\mu<1$. From (5.1), it can be seen that the fractional-order differentiation of $x_{1}$ is used to construct the sliding surface. Meanwhile, as $-1<\mu-1<0$, the operator $D^{\mu-1} x_{2}$ in (5.1), which means the $(\mu-1)$ th-order integration of $x_{2}$, can be seen as a low-pass filter and can reduce the amplitude of high-frequency fluctuations of $x_{2}$. In this sense, the fractional-order sliding surface defined by (5.1) is more smooth compared with the conventional sliding surface shown as (4.7).

\subsection{Design of FOSMC}

Taking the time derivative on both sides of (5.1) yields

$$
\dot{S}=k_{p} \dot{x}_{1}+k_{d} D^{\mu+1} x_{1}=k_{p} x_{2}+k_{d} D^{\mu-1} \dot{x}_{2}
$$

Substituting (4.4) into (5.2), we have

$$
\dot{S}=k_{p} x_{2}+k_{d} D^{\mu-1} \dot{x}_{2}=k_{p} x_{2}+k_{d} D^{\mu-1}\left\{\ddot{\theta}_{\text {ref }}-\frac{1}{J}\left[P \psi_{f} i_{q}-T_{L}-B \omega_{m}\right]\right\},
$$

when $T_{L}=0$, and forcing $\dot{S}=0$, then the control output can be obtained as follows:

$$
D^{\mu-1}\left\{\ddot{\theta}_{\text {ref }}-\frac{1}{J}\left[P \psi_{f} i_{q}-B \omega_{m}\right]\right\}=-\frac{k_{p}}{k_{d}} x_{2} .
$$

Taking the $(1-\mu)$ th-order derivative on both sides of (5.4) will result in

$$
\ddot{\theta}_{\mathrm{ref}}-\frac{1}{J}\left[P \psi_{f} i_{q}-B \omega_{m}\right]=D^{1-\mu}\left(-\frac{k_{p}}{k_{d}} x_{2}\right) .
$$

From (5.5), the equivalent control can be obtained as

$$
U_{\mathrm{eq}}=i_{q}=\frac{J}{P \psi_{f}}\left(\frac{k_{p}}{k_{d}} D^{1-\mu} x_{2}+\ddot{\theta}_{\mathrm{ref}}+\frac{1}{J} B \omega_{m}\right) .
$$

Similar to (4.11), when the system has load disturbances with upper limit $T_{L-\max }$, then the control output of FOSMC method can be given as

$$
U=i_{q}=U_{\mathrm{eq}}+k \operatorname{sgn}(S)=\frac{J}{P \psi_{f}}\left(\frac{k_{p}}{k_{d}} D^{1-\mu} x_{2}+\ddot{\theta}_{\mathrm{ref}}+\frac{1}{J} B \omega_{m}\right)+k \operatorname{sgn}(S),
$$

where $\mu$ is called as the order of FOSMC method. If we set $k_{p}=c, k_{d}=1$, and let $A=P \psi_{f} / J$, then the block diagram of the proposed FOSMC method can be shown in Figure 1. 


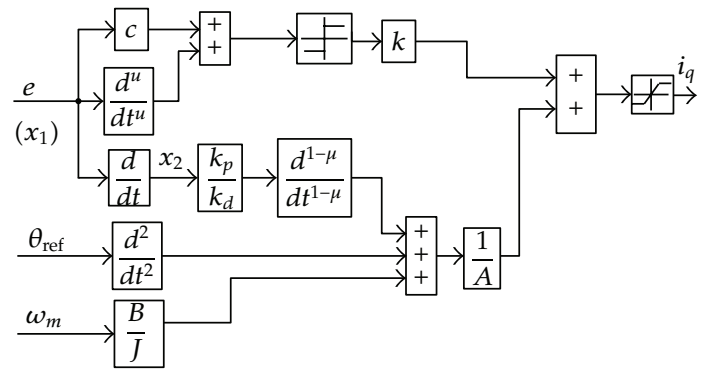

Figure 1: Block diagram of the proposed FOSMC method.

\subsection{Stability Analysis of FOSMC with Sign Function}

When the sign function is used in the control output, then substituting (5.7) into (5.3), we have

$$
\dot{S}=k_{d} D^{\mu-1}\left(\frac{T_{L-\max }}{J}-\frac{P \psi_{f}}{J} k \operatorname{sgn}(S)\right) .
$$

From (5.8), we can get the following.

(a) When $S<0$, then $\operatorname{sgn}(S)=-1$, and we have

$$
\delta_{1} \triangleq\left(\frac{T_{L-\max }}{J}-\frac{P \psi_{f}}{J} k \operatorname{sgn}(S)\right)=\left(\frac{T_{L-\max }}{J}+\frac{P \psi_{f}}{J} k\right)>0 .
$$

So the $(\mu-1)$ th-order fractional integration of $\delta_{1}$ is higher than zero, that is,

$$
\dot{S}=k_{d} D^{\mu-1}\left(\frac{T_{L-\max }}{J}-\frac{P \psi_{f}}{J} k \operatorname{sgn}(S)\right)>0,
$$

which implies that the derivative of the Lyapunov function $\dot{V}=S \dot{S}<0$.

(b) When $S>0$, then $\operatorname{sgn}(S)=1$, and we have

$$
\dot{S}=k_{d} D^{\mu-1}\left(\frac{T_{L-\max }}{J}-\frac{P \psi_{f}}{J} k\right) .
$$

From (5.11), it is clear that when

$$
\delta_{2} \triangleq \frac{T_{L-\max }}{J}-\frac{P \psi_{f}}{J} k<0,
$$

that is,

$$
k>\frac{T_{L-\max }}{P \psi_{f}},
$$


then the $(\mu-1)$ th-order fractional integration of $\delta_{2}$ is lower than zero, that is, $\dot{S}<0$, which means that $\dot{V}=S \dot{S}<0$.

From (5.8) to (5.13), it is obvious that when

$$
k>\frac{T_{L-\max }}{P \psi_{f}}
$$

then the system is globally stable; $S$ and $\dot{S}$ will approach zero in a finite time duration.

Moreover, from (5.8), it can be seen that because of the integration effect by the operator $D^{\mu-1}(\cdot)$, the variation amplitude of $\dot{S}$ in (5.8) is smaller than that of $\dot{S}$ in (4.14), which means that when the sign function is used, the sliding surface of the proposed FOSMC method has smaller chattering amplitude than the sliding surface of the conventional SMC method.

\subsection{Stability Analysis of FOSMC with Saturation Function}

From (5.7), it can be seen that the sign function is involved in the output, so the chattering phenomenon will be caused. In this paper, a saturation function is adopted to reduce the chattering problem, described as follows:

$$
\operatorname{sat}(S)= \begin{cases}1 & S>\varepsilon, \\ \frac{S}{\varepsilon} & -\varepsilon \leq S \leq \varepsilon, \\ -1 & S<-\varepsilon,\end{cases}
$$

where $\varepsilon>0$ denotes the thickness of the boundary layer.

When the saturation function is used, the control output can be rewritten as

$$
U=i_{q}=U_{\mathrm{eq}}+k \operatorname{sat}(S)=\frac{J}{P \psi_{f}}\left(\frac{k_{p}}{k_{d}} D^{1-\mu} x_{2}+\ddot{\theta}_{\mathrm{ref}}+\frac{1}{J} B \omega_{m}\right)+k \operatorname{sat}(S),
$$

then, similar to (5.8), substituting (5.16) into (5.3), we have

$$
\dot{S}=k_{d} D^{\mu-1}\left(\frac{T_{L-\max }}{J}-\frac{P \psi_{f}}{J} k \operatorname{sat}(\mathrm{S})\right)
$$

From (5.17), the following is clear.

(a) When $S<0$, then $\operatorname{sat}(S)<0$,

$$
\delta_{3} \triangleq\left(\frac{T_{L-\max }}{J}-\frac{P \psi_{f}}{J} k \operatorname{sat}(S)\right)>0 .
$$


So the $(\mu-1)$ th-order fractional integration of $\delta_{3}$ is higher than zero, that is,

$$
\dot{S}=k_{d} D^{\mu-1}\left(\frac{T_{L-\max }}{J}-\frac{P \psi_{f}}{J} k \operatorname{sat}(S)\right)>0,
$$

which means that the derivative of the Lyapunov function $\dot{V}=S \dot{S}<0$.

(b) When $S>\varepsilon$, then $\operatorname{sat}(S)=1$, and we have

$$
\dot{S}=k_{d} D^{\mu-1}\left(\frac{T_{L-\max }}{J}-\frac{P \psi_{f}}{J} k\right) .
$$

Similar with (5.11)-(5.13), when

$$
k>\frac{T_{L-\max }}{P \psi_{f}},
$$

then $\dot{S}<0$, which means that $\dot{V}=S \dot{S}<0$.

(c) When $0<S \leq \varepsilon$, then $\operatorname{sat}(S)=S / \varepsilon$, and we have

$$
\dot{S}=k_{d} D^{\mu-1}\left(\frac{T_{L-\max }}{J}-\frac{P \psi_{f}}{J} k \frac{S}{\varepsilon}\right) .
$$

From (5.22), it can be seen that when

$$
\delta_{4} \triangleq\left(\frac{T_{L-\max }}{J}-\frac{P \psi_{f}}{J} k \frac{S}{\varepsilon}\right)<0,
$$

that is,

$$
k>\frac{T_{L-\max }}{P \psi_{f}} \frac{\varepsilon}{S} \geq \frac{T_{L-\max }}{P \psi_{f}}
$$

then $\dot{S}<0$. Here, it is assumed that a load disturbance with magnitude $T_{L \text {-max }}$ is exerted on the system. From (5.24), it can be seen that when the value of $S$ is very small, then $\varepsilon / S \gg 1$, so the condition for $\dot{\mathrm{S}}<0$ is that the value of $k$ is much higher than $T_{L-\max } / P \psi_{f}$, but in fact the parameter $k$ will not be given a so high value. Here, it is assumed that $k$ is assigned a minimum value which meets condition (5.21). Then the sliding surface $S$ will undergo the following stages.

(i) In the period $0<S \ll \varepsilon$, we have $\dot{S} \gg 0$, so the system is unstable, meanwhile $S$ will rapidly arrive at the peak value $S^{*}\left(\right.$ where $S^{*}>\varepsilon$ ) in a finite time with large initial positive velocity.

(ii) As $S=S^{*}>\varepsilon$, then from (5.24), it can be seen that the value of $k$ satisfies condition (5.21), so $\dot{S}<0$ and $\dot{V}=S \dot{S}<0$, that is, the system is globally stable again. In this moment, because $S>0$ and $\dot{S}<0$, then $S$ will decrease with negative velocity. 
(iii) When $S$ decreases until $S<\varepsilon$, then from (5.24), it can be seen that the value of $k$ does not satisfy condition (5.21) any longer, which means that $\dot{S}>0$, then $S$ starts to increase.

(iv) When $S$ increases until $S>\varepsilon$, then similar to (ii), we have $S>0$ and $\dot{S}<0$, then $S$ will decrease with negative velocity.

(v) After several oscillations and adjustments between stages (iii) and (iv), the sliding surface function $S$ will finally maintain on the point of $S=\varepsilon$, and the system is in a stable state with $\dot{S}=0$.

When the system is in the stable state described by (v), then from (4.7) or (5.1), it can be seen that $x_{2}=0$ or $D^{\mu} x_{1}=0$, and the stable position error $x_{1}$ can be estimated as follows:

$$
S=c x_{1}=\varepsilon \Longrightarrow x_{1}=\frac{\varepsilon}{c}
$$

or

$$
S=k_{p} x_{1}=\varepsilon \Longrightarrow x_{1}=\frac{\varepsilon}{k_{p}}
$$

Generally, when the load disturbance is $T_{L}\left(T_{L}<T_{L-\max }\right)$, then similar to the above analysis, the stable position error $x_{1}$ can be estimated as follows:

$$
S=c x_{1}=\frac{\varepsilon T_{L}}{k P \psi_{f}} \Longrightarrow x_{1}=\frac{\varepsilon T_{L}}{c k P \psi_{f}}
$$

or

$$
S=k_{p} x_{1}=\frac{\varepsilon T_{L}}{k P \psi_{f}} \Longrightarrow x_{1}=\frac{\varepsilon T_{L}}{k_{p} k P \psi_{f}} .
$$

With the maximum permissible position error $x_{1}$ of the PMSM system, (5.26) or (5.28) will be the constraint in designing the parameter $\varepsilon$ and $c$ or $k_{p}$.

Remark 5.1. In the above analysis of parts (b) and (c), the integration effect of the operator $D^{\mu-1}(\cdot)$ is ignored temporarily. If the integration effect is considered, then the fractional-order $\mu$ will decide the phase delay and variation magnitude of $\dot{S}$. When $\mu$ is too small, especially when $\mu=0$, then the operator $D^{\mu-1}(\cdot)$ becomes a first-order integer integrator, and the long time integration effect will lead to the largest phase delay and smallest variation magnitude of $\dot{S}$, and the stable condition $\dot{V}=S \dot{S}<0$ may not be satisfied promptly, and so the system will become unstable. When $\mu$ is too large, especially when $\mu=1$, then the operator $D^{\mu-1}(\cdot)$ does not have integration action, and $\dot{S}$ has zero-phase delay and the largest variation magnitude, which are the same as the convention SMC method. When $\mu$ is selected as a proper value in the range $(0,1)$, then the suitable phase delay of $\dot{S}$ will satisfy the stable condition $\dot{V}=S \dot{S}<0$, and meanwhile, the appropriate variation magnitude of $\dot{S}$ will make the sliding surface $S$ change with small fluctuation, so a better control performance can be obtained. 


\section{Robustness and Effectiveness Analysis of FOSMC}

The robustness and effectiveness of the proposed FOSMC method will be analyzed in the following two aspects.

\subsection{Analysis of the Control Output}

From the control output of the FOSMC method shown as (5.7) or (5.16), it can be seen that two important terms are included.

(a) The term $D^{1-\mu} x_{2}$ denotes the $(1-\mu)$ th-order differentiation of $x_{2}$, so the fractional dimension accelerating change rate of position error is contained in the output, which means that the output of the FOSMC method is more sensitive to the change rate of position error and can provide a prompt output.

(b) The other term is the $\operatorname{sgn}(S)$ in (5.7) or the $\operatorname{sat}(S)$ in (5.16), the former is a high-frequency switching signal, and the latter is a relative smooth switch signal. According to the sliding surface $S$ defined by (5.1), it is clear that an $(\mu-1)$ th-order integrator for $x_{2}$ is contained, that is, the proposed sliding surface $S$ is more smooth than the conventional sliding surface. In other words, by using the FOSMC method, the chattering of $\operatorname{sgn}(S)$ in (5.7) is eliminated to some degree, and the term sat $(S)$ in (5.16) is more smooth.

\subsection{Analysis of Stable Condition}

With (5.8) and (5.17), it can be seen that when substituting the control output into the derivative of fractional-order sliding surface $S$, we have

$$
\dot{S}=k_{d} D^{\mu-1}\left(\frac{T_{L-\max }}{J}-\frac{P \psi_{f}}{J} k \operatorname{sgn}(S)\right)=k_{d} D^{\mu-1} \delta_{1}
$$

or

$$
S=k_{d} D^{\mu-1}\left(\frac{T_{L-\max }}{J}-\frac{P \psi_{f}}{J} k \operatorname{sat}(S)\right)=k_{d} D^{\mu-1} \delta_{3}
$$

Here, the operator $D^{\mu-1}(\cdot)$ means the fractional-order integration since $0<\mu<1$.

The following is assumed:

(i) the value of $k$ is set as a constant which is satisfied with condition (5.14) or (5.21);

(ii) the system is in a reaching state (i.e., $\dot{V}=S \dot{S}<0$ ) or in a stable state (i.e., $S=$ 0 or constant, and $\dot{S}=0$ ).

Then the following three cases will be discussed.

(a) When the system is in a reaching state and $S>0, \dot{S}<0$, then

$$
\dot{S}=k_{d} D^{\mu-1} \delta_{1}<0 \Longrightarrow \delta_{1}<0
$$


or

$$
\dot{S}=k_{d} D^{\mu-1} \delta_{3}<0 \Longrightarrow \delta_{3}<0 .
$$

If an instant load disturbance $T_{\text {instant }}$ which is greater than $T_{L-\max }$ is applied on the system, then from (6.1) or (6.2), it can be seen that in this moment $\delta_{1}>0$ or $\delta_{3}>0$, but because of the integration effect by the fractional-order integration operator $D^{\mu-1}(\cdot)$, the integration value that is, $D^{\mu-1}\left(\delta_{1}\right)$ or $D^{\mu-1}\left(\delta_{3}\right)$, will not be greater than zero instantaneous, in other words the system will remain stable for an extra short time.

While for the conventional SMC method, from (4.14), it can be seen that the derivative of sliding surface $S$ is

$$
\dot{S}=\left[\frac{T_{L-\max }}{J}-\frac{P \psi_{f}}{J} k \operatorname{sgn}(S)\right]
$$

or

$$
\dot{S}=\left[\frac{T_{L-\max }}{J}-\frac{P \psi_{f}}{J} k \operatorname{sat}(S)\right]
$$

It is clear that when an instant load disturbance $T_{\text {instant }}\left(T_{\text {instant }}>T_{L \text {-max }}\right)$ is applied on the system, then $S<0$ immediately, and the system is also unstable at once.

(b) When the system is in a reaching state and $S<0, \dot{S}>0$, then

$$
\dot{S}=k_{d} D^{\mu-1} \delta_{1}>0 \Longrightarrow \delta_{1}>0
$$

or

$$
\dot{S}=k_{d} D^{\mu-1} \delta_{3}>0 \Longrightarrow \delta_{3}>0
$$

Similar to the above analysis, when an instant negative load disturbance (i.e., an opposite direction load disturbance) $T_{\text {instant }}$ which is smaller than $\left(-T_{L-\max }\right)$ is applied on the system, then from (6.1) or (6.2), it can be seen that in this moment $\delta_{1}>0$ or $\delta_{3}>0$, but because of the integration effect by the operator $D^{\mu-1}(\cdot)$, the integration value, that is, $D^{\mu-1}\left(\delta_{1}\right)$ or $D^{\mu-1}\left(\delta_{3}\right)$, will not be smaller than zero instantaneously in other words, the system will continue to be stable for an extra short time.

While for the conventional SMC method, it is clear that when an instant negative load disturbance $T_{\text {instant }}\left(T_{\text {instant }}<-T_{L-\max }\right)$ is exerted on the system, then according to (6.5) and (6.6), it can be seen that $\dot{S}$ will be smaller than zero (i.e., $\dot{S}<0$ ) immediately, and thus, the system is also unstable at once.

(c) When the system is in a stable state, that is, $S=0$ or constant, and $\dot{S}=0$, then

$$
\dot{S}=k_{d} D^{\mu-1} \delta_{1}=0 \Longrightarrow \delta_{1}=0
$$


or

$$
\dot{S}=k_{d} D^{\mu-1} \delta_{3}=0 \Longrightarrow \delta_{3}=0
$$

If an instant positive or negative load disturbance $T_{\text {instant }}$ is applied on the system, then from (6.1) or (6.2), it is obvious that in this moment there is a step change for $\delta_{1}$ or $\delta_{3}$, but because of the integration effect by the fractional-order integration operator $D^{\mu-1}(\cdot)$, the integration value, that is, $D^{\mu-1}\left(\delta_{1}\right)$ or $\mathrm{D}^{\mu-1}\left(\delta_{3}\right)$, will not change greatly in a short time, which means that the sliding surface $S$ will change with smaller fluctuation comparing with the conventional SMC method, so a better control performance is obtained.

In addition, when the load disturbance $T_{\text {instant }}$ is greater than $T_{L-\max }$, then the same conclusions as those made from the above analysis of (a) and (b) can be obtained.

From the above analysis, it is obvious that the proposed FOSMC method is more robust than the conventional SMC method.

\section{Numerical Computation Examples and Simulation}

\subsection{Approximation of Fractional-Order Operator}

The Matlab/Simulink is used to simulate the FOSMC control system. In the simulation, a discrete-time finite-dimensional $(z)$ transfer function is computed to approximate the continuous-time fractional-order operator $D^{\mu}(\cdot)$ by the IRID method [37], that is, dfod = irid_fod $\left(u, T_{s}, N\right)$. In the simulation, the sampling frequency of FOSMC controller is $2 \mathrm{KHz}$; thus, in the IRID method, $T_{s}=0.0005 \mathrm{sec}$, and the approximation order is $N=5$.

\subsection{System Block and Configuration}

The block diagram of the PMSM drive system using FOSMC method is shown in Figure 2, in which the block "SMC" means the conventional integer-order SMC method, and the block "FOSMC" is the proposed method, which is shown in Figure 1. The performance of the proposed FOSMC is compared with that of the conventional SMC. The rotor of the PMSM is the permanent magnet, and the flux linkage is constant. The specifications of the PMSM are shown in Table 1.

As shown in Figure 2, the drive system has an outer loop of position controller based on FOSMC method and an inner loop including two current controllers, that is, the $q$-axis and $d$-axis stator current regulators, both of which are based on PI control algorithm with sampling frequency of $10 \mathrm{KHz}$, and the $d$-axis stator current command is set to zero. In the block, $\omega_{\text {ref }}$ is the reference rotor speed in mechanical revolutions per minute, $\omega$ is the rotor speed in mechanical revolutions per minute measured by encoder, and the space vector PWM was used for the PWM generation.

For comparison, we first determine the optimal parameters of the conventional SMC method, and then the corresponding parameters of the new proposed FOSMC method are set similarely, that is, in Figure 2, the following parameters of SMC and FOSMC are set to be the same, that is,

$$
k=3, \quad k_{p}=c=100, \quad k_{d}=1, \quad \varepsilon=1 .
$$




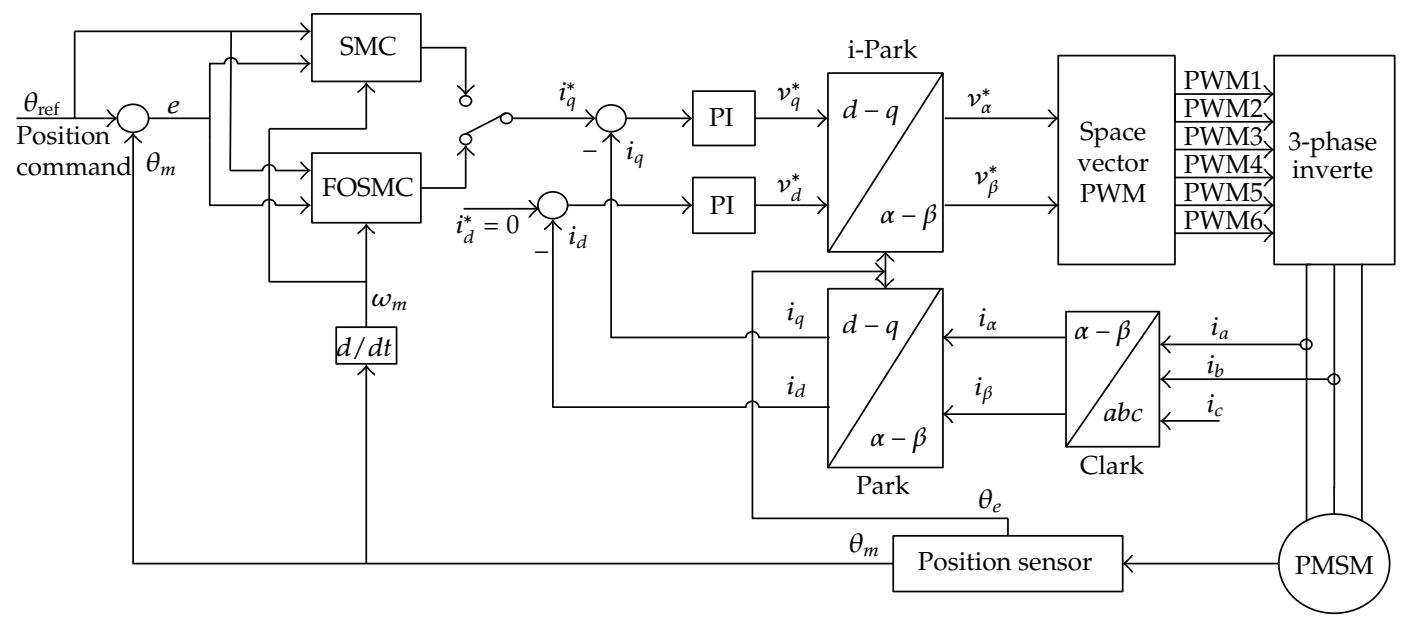

Figure 2: Block diagram of the PMSM position control system.

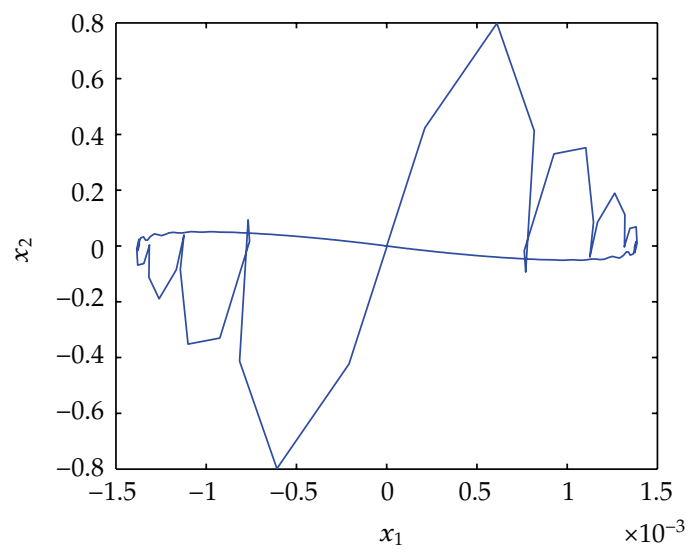

(a) Phase trace of the conventional SMC method

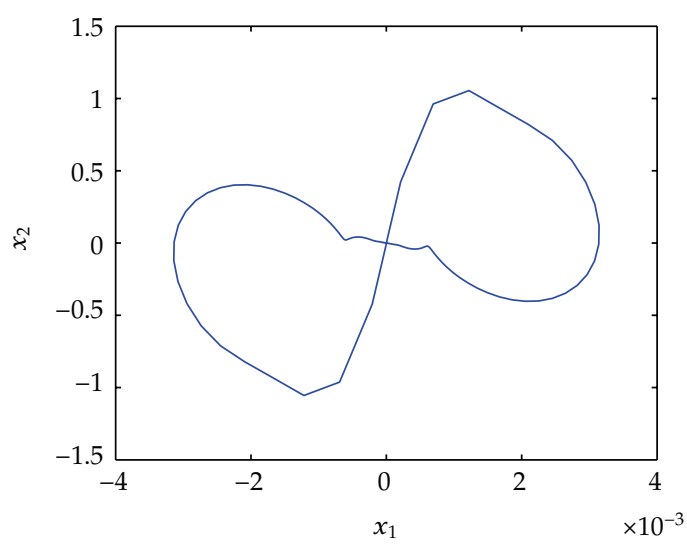

(b) Phase trace of the proposed FOSMC method $(\mu=0.6)$

Figure 3: Phase traces of the conventional SMC method and the proposed FOSMC method with saturation function.

\subsection{Simulation and Comparison}

\subsubsection{Simulation of Phase Trace}

In this simulation, the phase traces by the conventional SMC method and the proposed FOSMC method are simulated and compared. The given position reference is $\theta_{\text {ref }}=\pi \mathrm{rad}$, which is a step input with soft-start mode, and the order of the proposed FOSMC method is $\mu=0.6$.

Figure 3 shows the simulation results of the phase traces by the conventional SMC method and the proposed FOSMC method with saturation function. Figure 4 is similar to Figure 3 , and the only difference is that the saturation function is replaced by the sign function in the two methods. 


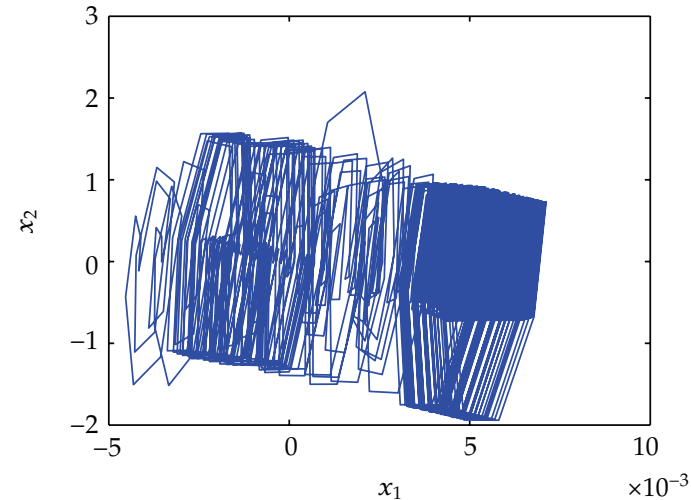

(a) Phase trace of conventional SMC method

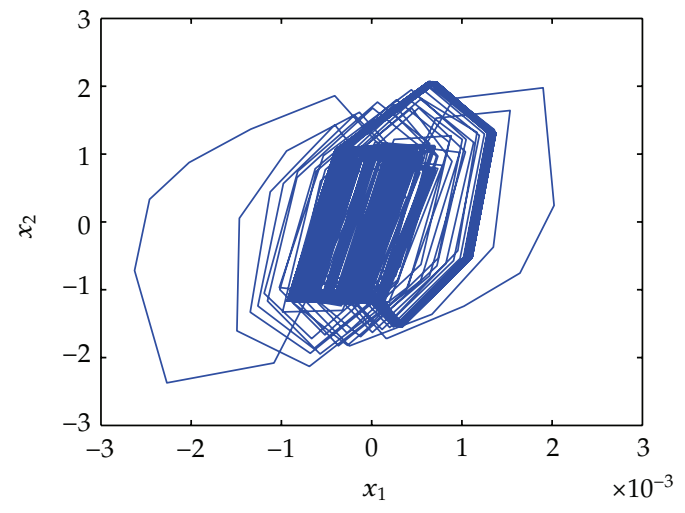

(b) Phase trace of the proposed FOSMC method $(\mu=0.6)$

Figure 4: Phase traces of the conventional SMC method and the proposed FOSMC method with sign function.

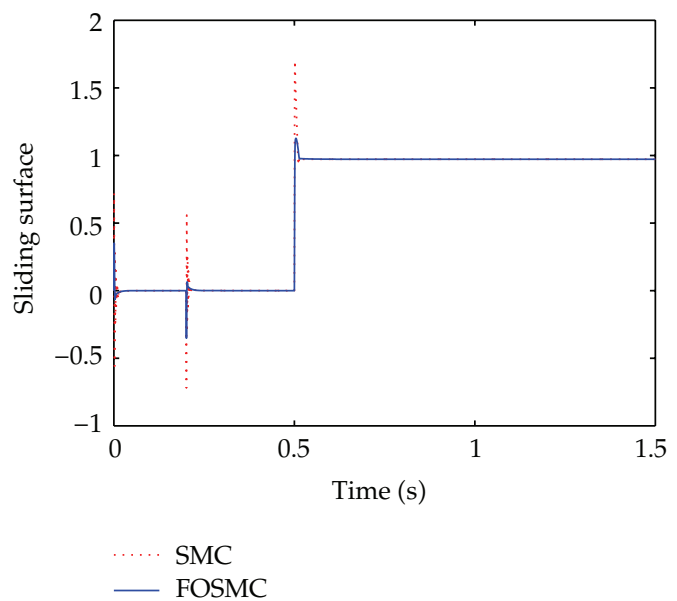

Figure 5: Sliding surfaces with saturation function and load disturbance of $2.5 \mathrm{Nm}$ at $t=0.5 \mathrm{~s}(\mu=0.6)$.

From Figure 3, it can be seen that the phase traces of both methods can reach the sliding surface $(S=0)$ and arrive at the origin finally, but because of the fractional-order integration effect (i.e., the term $D^{\mu-1} x_{2}$ in $S$ ), the phase trace of the proposed FOSMC method is more smooth than that of the conventional SMC method; this also means that the proposed FOSMC has smaller speed vibration, which is consistent with the analysis of Section 5.1.

From Figure 4, it is obvious that the phase trace of the proposed FOSMC method is more focused on the origin than that of the conventional SMC method, which means that the proposed FOSMC has smaller speed error.

\subsubsection{Simulation of Stability Condition}

In this simulation, the stability condition will be tested. The position reference is step input $\theta_{\text {ref }}=\pi \mathrm{rad}$, the order of FOSMC is $\mu=0.6$, and other parameters are set as (7.1). From 


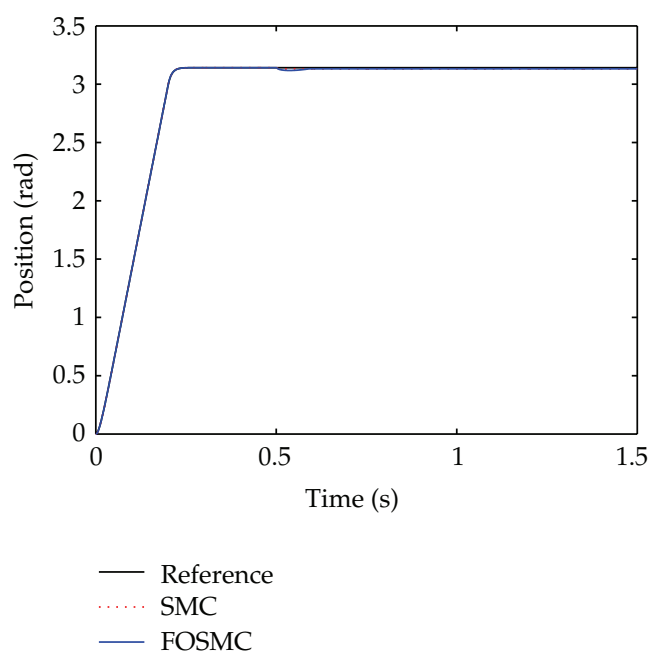

(a) Position responses

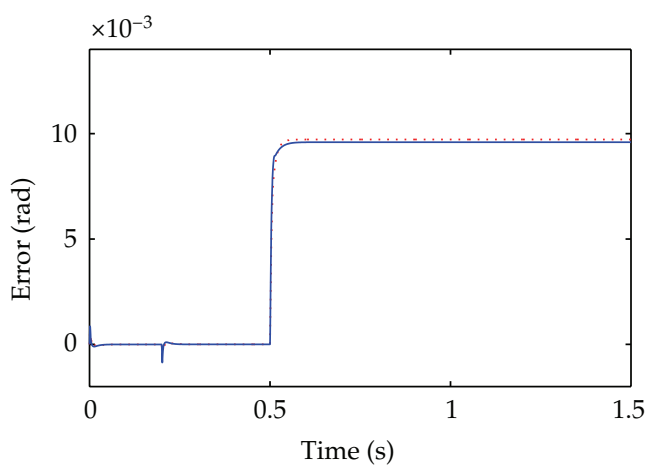

SMC

FOSMC

(b) Position error

Figure 6: Position responses and error with saturation function and load disturbance of $2.5 \mathrm{Nm}$ at $t=0.5 \mathrm{~s}$ $(\mu=0.6)$.

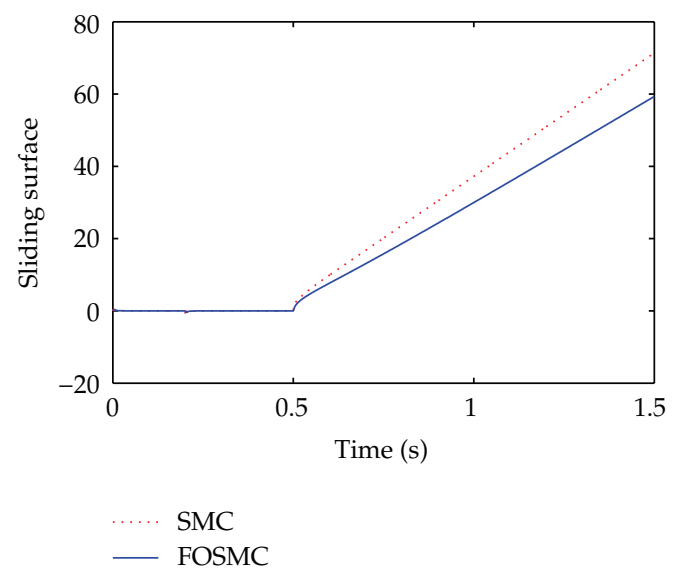

Figure 7: Sliding surfaces with saturation function and load disturbance of $2.6 \mathrm{Nm}$ at $t=0.5 \mathrm{~s}(\mu=0.6)$.

(5.13), (5.21), and Table 1, it can be calculated that the maximum load disturbance is $T_{L-\max }=$ $2.568 \mathrm{Nm}$. In each of the following cases, the conventional SMC method and the proposed FOSMC method are executed.

Figures 5-8 are the time curves of sliding surface function $S$, position responses, and position error, respectively. The saturation function is adopted, and different load disturbance is applied at time $t=0.5 \mathrm{~s}$.

In Figures 5 and 6 , the load disturbance is $2.5 \mathrm{Nm}$, and we can see that the system controlled by SMC or FOSMC is stable, because the load disturbance is less than $T_{L \text {-max }}$. Meanwhile, from Figures 5 and $6(\mathrm{~b})$, it can be seen that the stable value of sliding surface function is $S \approx \varepsilon=1$, and the stable position error is $x_{1} \approx \varepsilon / c=0.01$, which are consistent with the analysis of Section 5.4 and (5.26). 


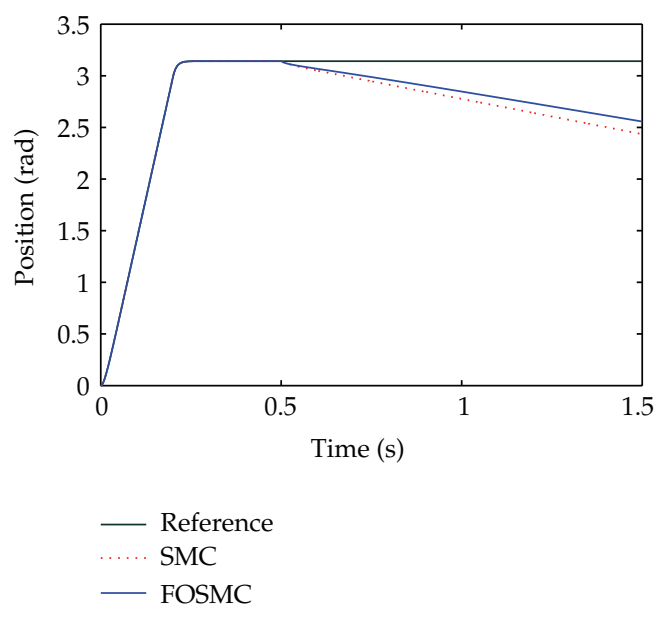

(a) Position responses

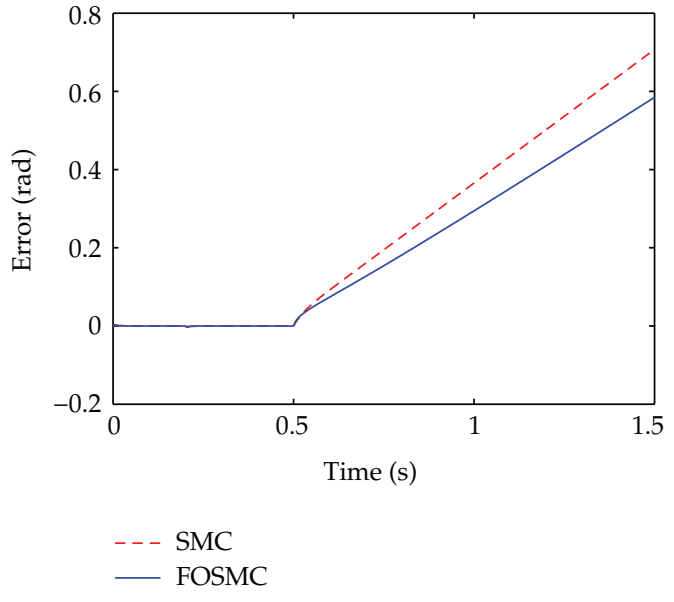

(b) Position error

Figure 8: Position responses and error with saturation function and load disturbance of $2.6 \mathrm{Nm}$ at $t=0.5 \mathrm{~s}$ $(\mu=0.6)$.

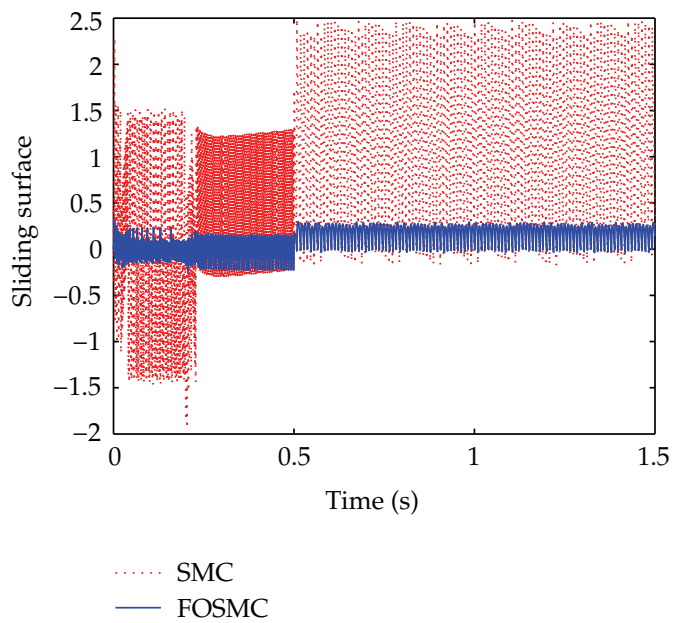

Figure 9: Sliding surfaces with sign function and load disturbance of $2.3 \mathrm{Nm}$ at $t=0.5 \mathrm{~s}(\mu=0.6)$.

From Figure 5, one can see that when the external load is exerted on the system at $t=0.5 \mathrm{~s}$, the variation amplitude of the sliding surface by the FOSMC method is smaller than that of the conventional SMC method, and consequently, the position error by the FOSMC method is smaller than that by the conventional SMC method, just as shown by Figure 6(b). The above two simulation results meet the analysis of Section 6.2(c).

In Figures 7 and 8 , the load disturbance is $2.6 \mathrm{Nm}$, and it is obvious that the system controlled by SMC or FOSMC method is unstable, just because the load disturbance is greater than $T_{L \text {-max }}$. Moreover, an important result can be obtained from Figures 7 and 8 , that is, when the load disturbance is greater than $T_{L \text {-max }}$, although the system is unstable any longer, the 


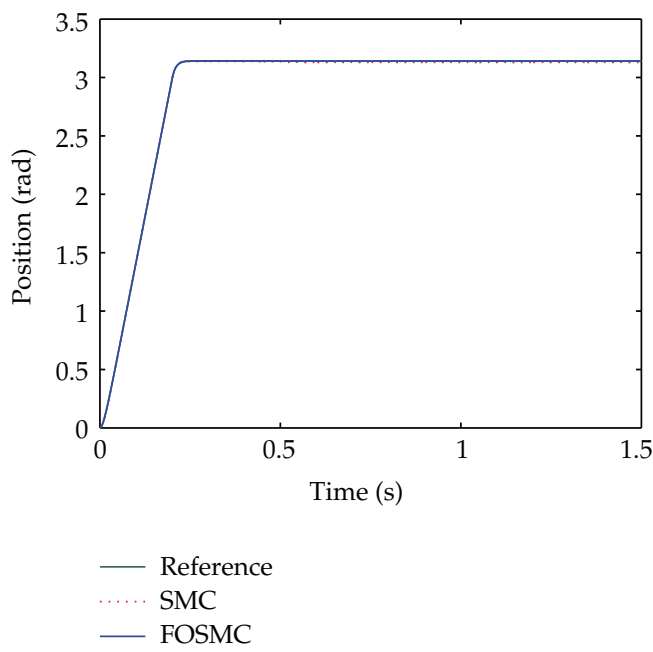

(a) Position responses

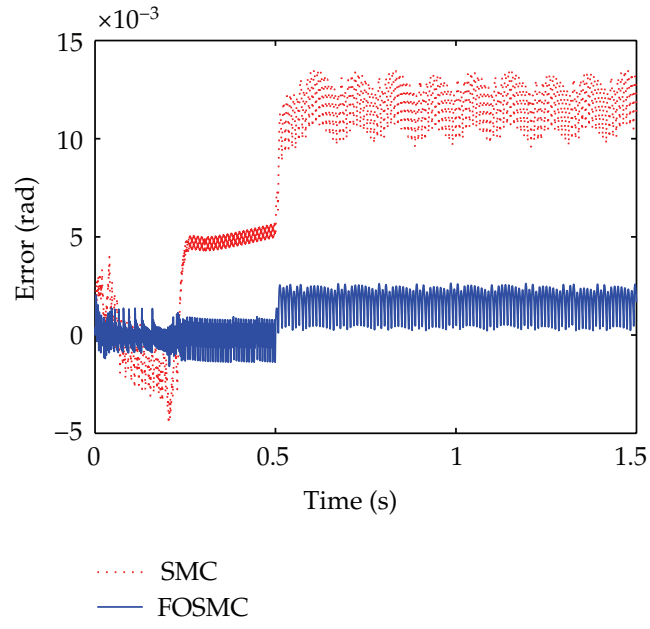

(b) Position error

Figure 10: Position responses and error with sign function and load disturbance of $2.3 \mathrm{Nm}$ at $t=0.5 \mathrm{~s}$ $(\mu=0.6)$.

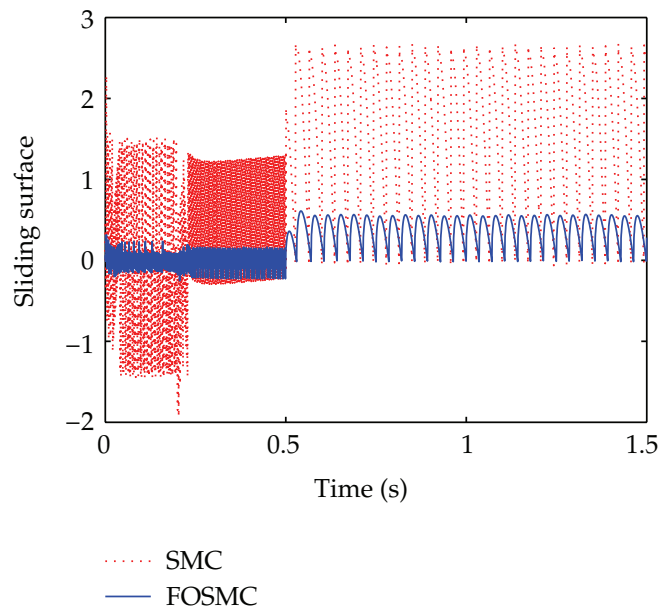

Figure 11: Sliding surfaces with sign function and load disturbance of $2.5 \mathrm{Nm}$ at $t=0.5 \mathrm{~s}(\mu=0.6)$.

position error by the proposed FOSMC method is smaller than that by the conventional SMC method, which is keeping with the analysis of Section 6.2.

Figures 9-14 are the time curves of sliding surface function $S$, position responses, and position error, respectively, in which the sign function is adopted, and different load disturbance is applied at $t=0.5 \mathrm{~s}$.

In Figures 9 and 10, the load disturbance is $2.3 \mathrm{Nm}$, and we can see that the system is stable under the load disturbance, because the load disturbance is less than $T_{L \text {-max }}$. Because of the use of sign function, the chattering phenomenon exists in the sliding surface $S$, just as shown in Figure 9. Meanwhile from Figures 9 and 10, two important results can be seen, that is, (a) the chattering amplitude of the sliding surface $S$ by the FOSMC method is smaller 


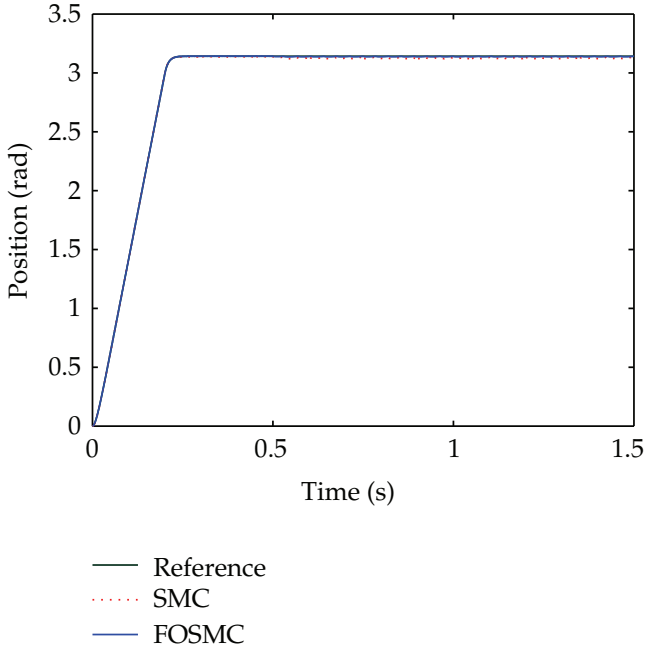

(a) Position responses

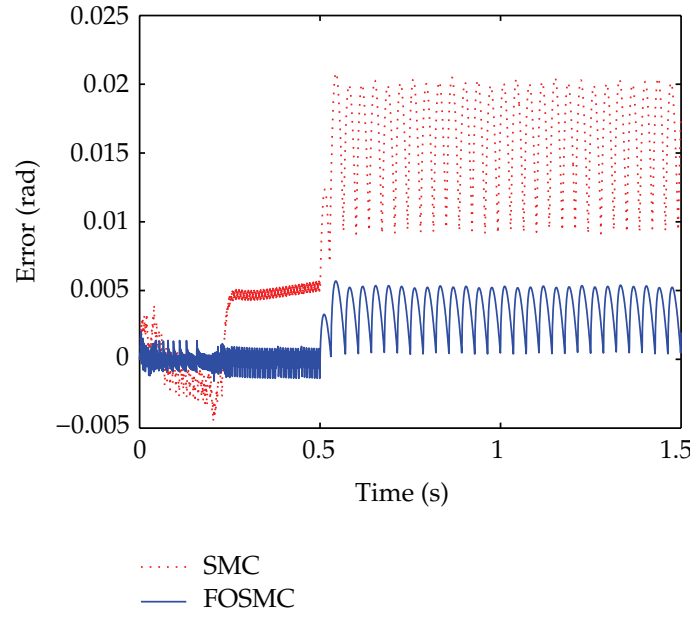

(b) Position error

Figure 12: Position responses and error with sign function and load disturbance of $2.5 \mathrm{Nm}$ at $t=0.5 \mathrm{~s}$ $(\mu=0.6)$.

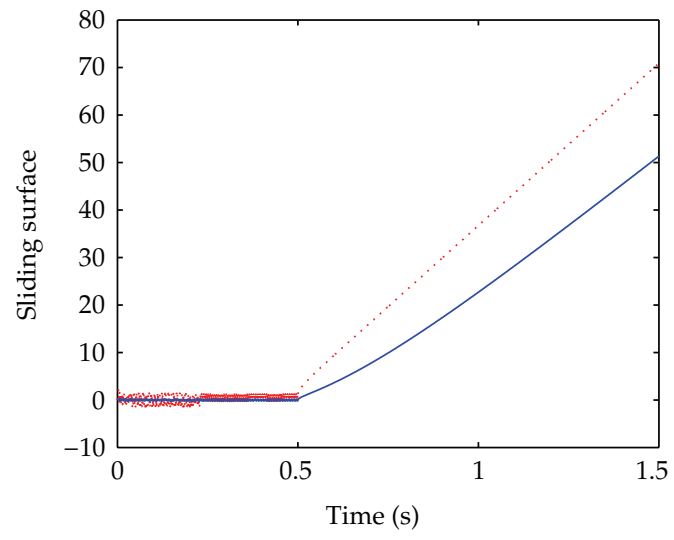

$\mathrm{SMC}$

FOSMC

Figure 13: Sliding surfaces with sign function and load disturbance of $2.6 \mathrm{Nm}$ at $t=0.5 \mathrm{~s}(\mu=0.6)$.

than that by the conventional SMC method; (b) the position error by the proposed FOSMC method is also distinctly smaller than that by the conventional SMC method. The above two results meet the analysis of Sections 5.3 and 6.1 .

In Figures 11 and 12, the load disturbance is $2.5 \mathrm{Nm}$, and it can be seen that the system is critically stable after the load disturbance is applied, just because the load disturbance is close to $T_{L-\max }$. And we also can see that the chattering amplitude of the sliding surface $S$ and the position error, by the FOSMC method, are also distinctly smaller than those by the conventional SMC method, which meet the analysis of Sections 5.3 and 6.1. 


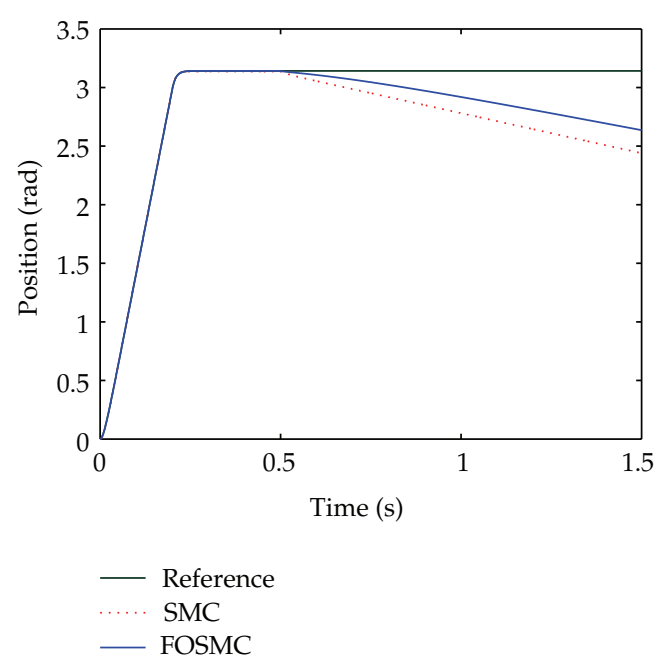

(a) Position responses

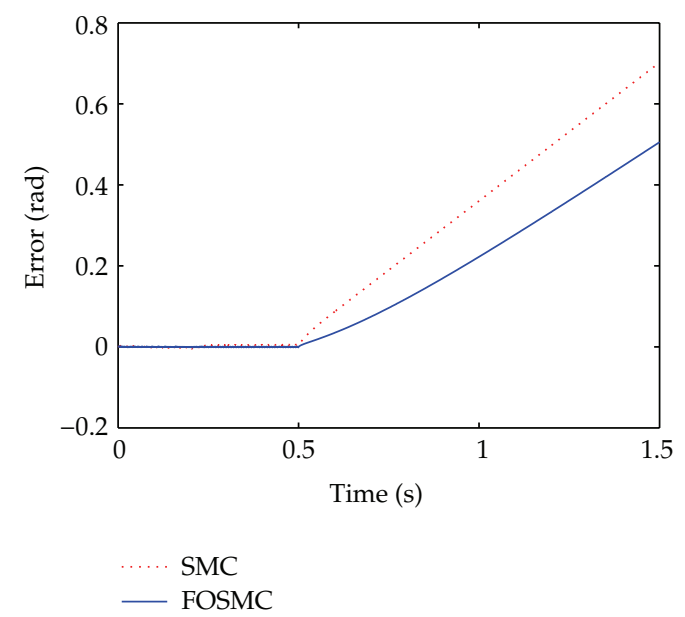

(b) Position error

Figure 14: Position responses with sign function and load disturbance of $2.6 \mathrm{Nm}$ at $t=0.5 \mathrm{~s}(\mu=0.6)$.

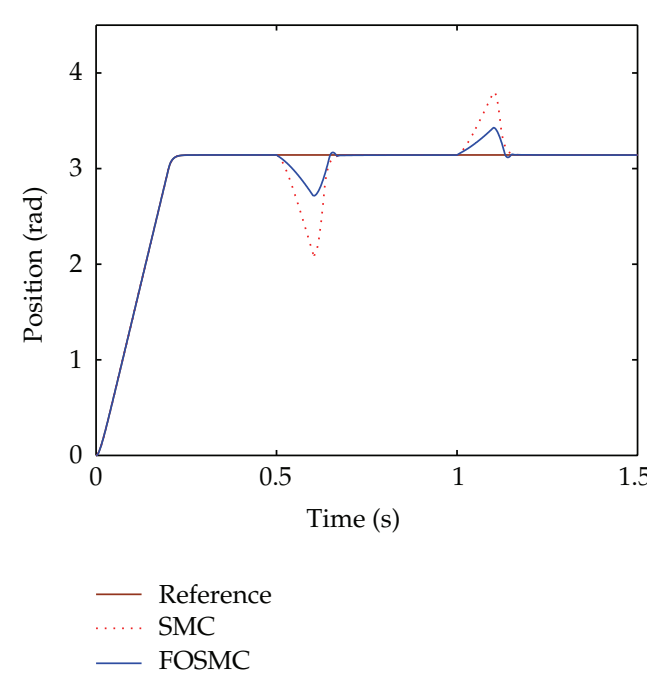

(a) Position responses

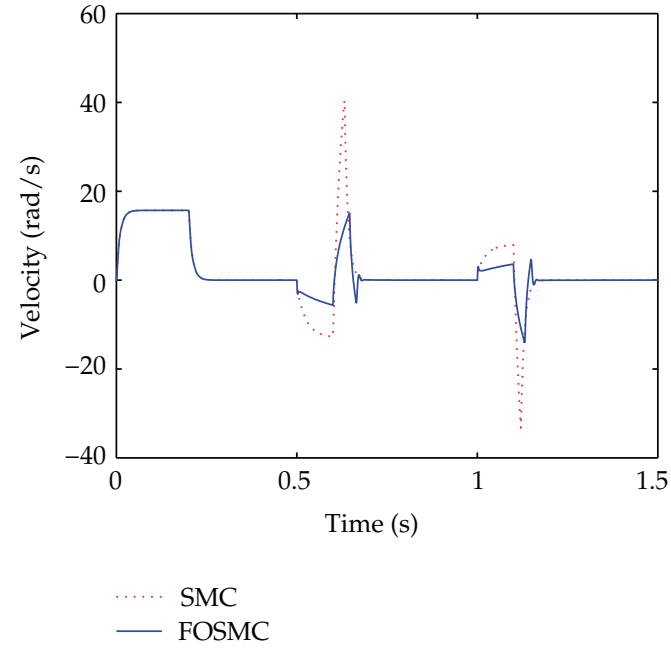

(b) Velocity responses

Figure 15: Position responses and velocity responses with load disturbances around $t=0.5 \mathrm{~s}$ and $1.0 \mathrm{~s}$.

In Figures 13 and 14, the load disturbance is $2.6 \mathrm{Nm}$, and it is clear that the system driven by FOSMC or SMC method is unstable after the time $0.5 \mathrm{~s}$, just because the load disturbance is greater than $T_{L-\max }$. Meanwhile, although the system is unstable any longer, the position error by the proposed FOSMC method is smaller than that by the conventional SMC method, which is keeping with the analysis of Section 6.2.

All of the above simulation results show the correctness of the stability condition shown by (5.13) or (5.21); meanwhile, the robustness analyses of Sections 5.3, 5.4, and 6 are also verified. 


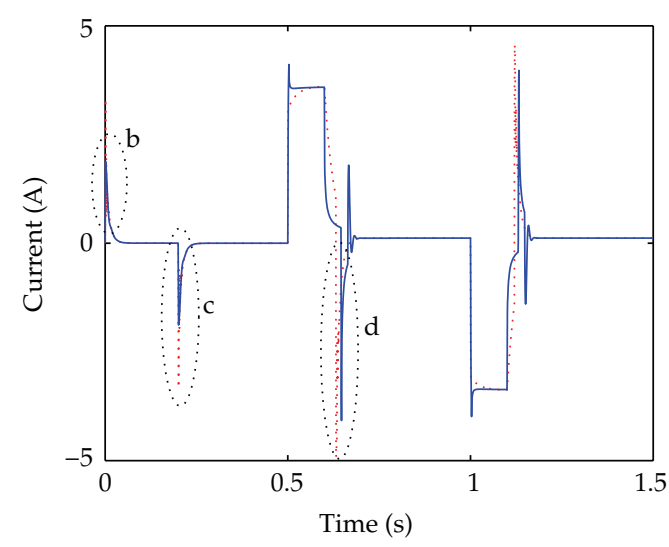

… SMC

- FOSMC

(a) Control outputs with load disturbances around $t=$ $0.5 \mathrm{~s}$ and $1.0 \mathrm{~s}$

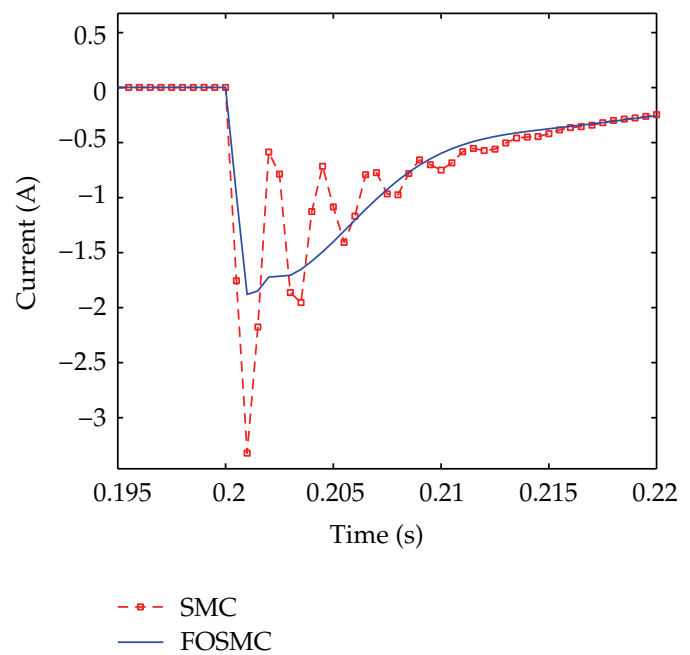

(c) Control outputs during the time $[0.195 \mathrm{~s}, 0.22 \mathrm{~s}]$

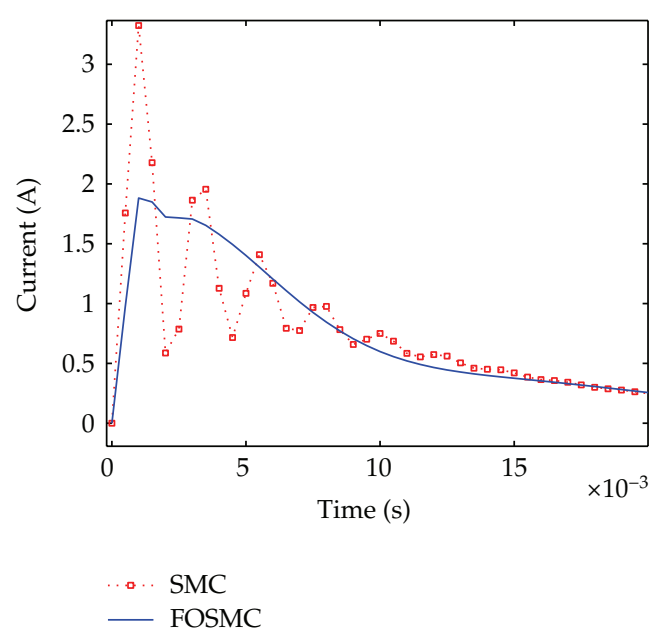

(b) Control outputs during the time [0s, $0.02 \mathrm{~s}]$

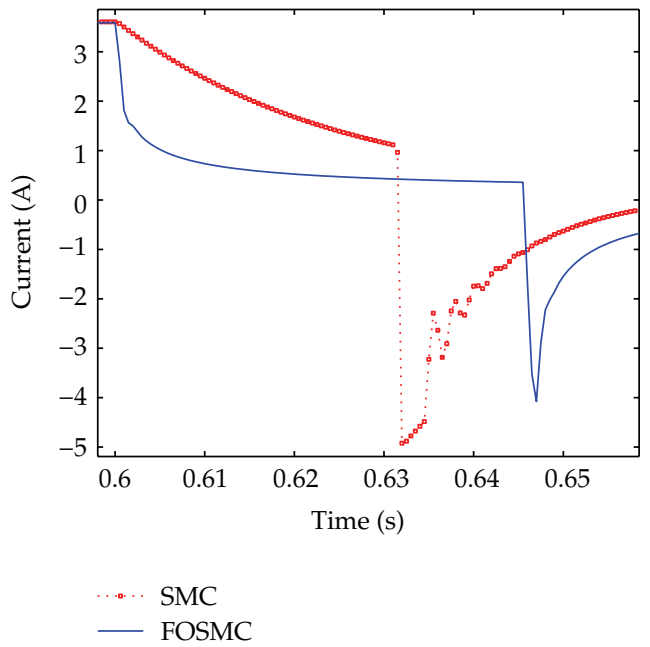

(d) Control outputs during the time $[0.60 \mathrm{~s}, 0.66 \mathrm{~s}]$

Figure 16: Results of the control outputs.

\subsubsection{Simulation of Dynamic Position Response with Step Input Signal}

In this simulation, the position reference is $\theta_{\text {ref }}=\pi \mathrm{rad}$, the order of FOSMC is $\mu=0.6$, and the saturation function is adopted. A step disturbance load of $3.1 \mathrm{Nm}$ is applied at $t=0.5 \mathrm{~s}$ and withdrawn at $t=0.6 \mathrm{~s}$, another step disturbance load of $-3.1 \mathrm{Nm}$ is applied at $t=1.0 \mathrm{~s}$ and withdrawn at $t=1.1 \mathrm{~s}$. Figures $15(\mathrm{a})$ and $15(\mathrm{~b})$ show the dynamic position and velocity responses, respectively, of the conventional SMC method and the proposed FOSMC method in the presence of the above disturbances load. Obviously, the position error by the proposed FOSMC method is significantly smaller than that by the conventional SMC method; in other words, the FOSMC method is of more robustness than the conventional SMC method, which is in agreement with the analysis of Section 6. 


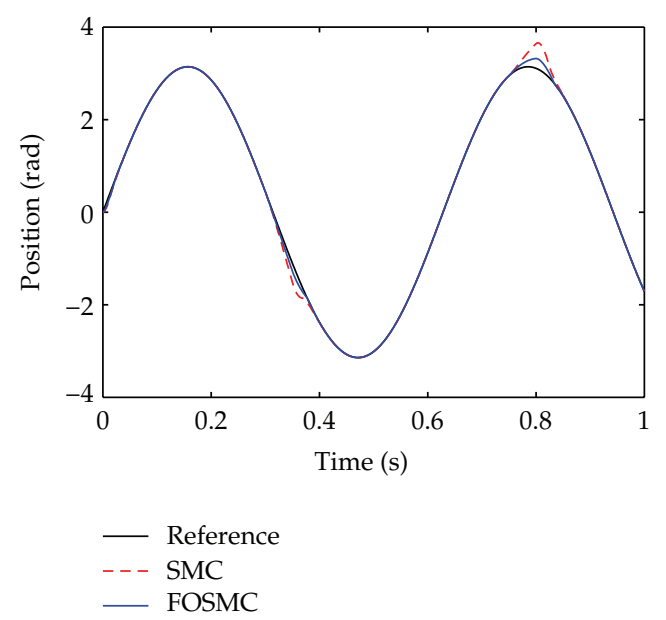

(a) Position responses

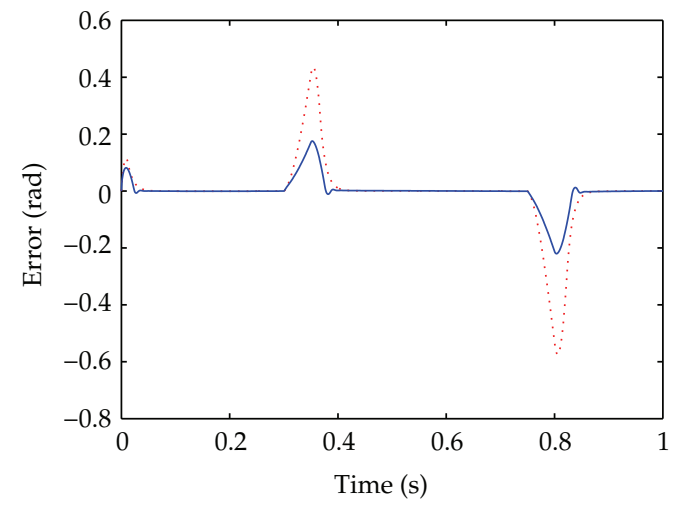

… SMC

- FOSMC

(b) Position error

Figure 17: Position responses and error to sinusoidal input signal with load disturbances at $t=0.3 \mathrm{~s}$ and $0.75 \mathrm{~s}$.

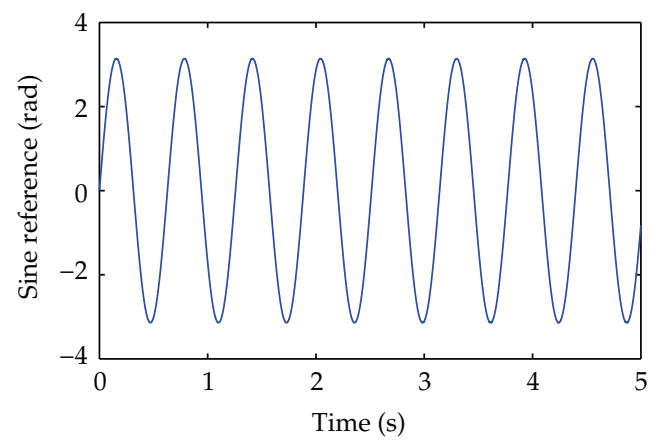

Figure 18: Sine reference input.

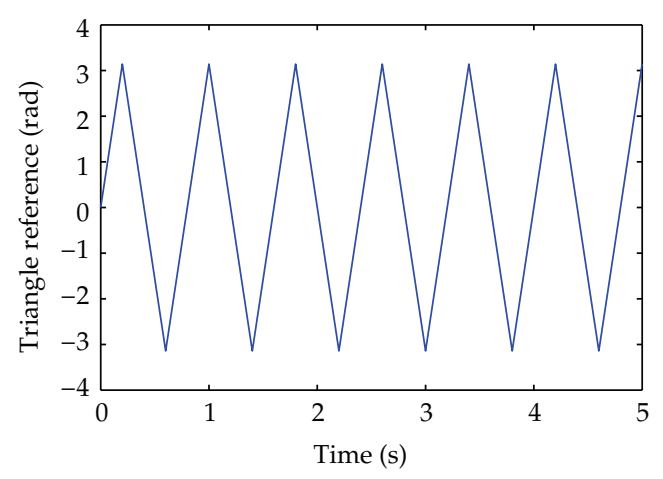

Figure 19: Triangle reference input. 


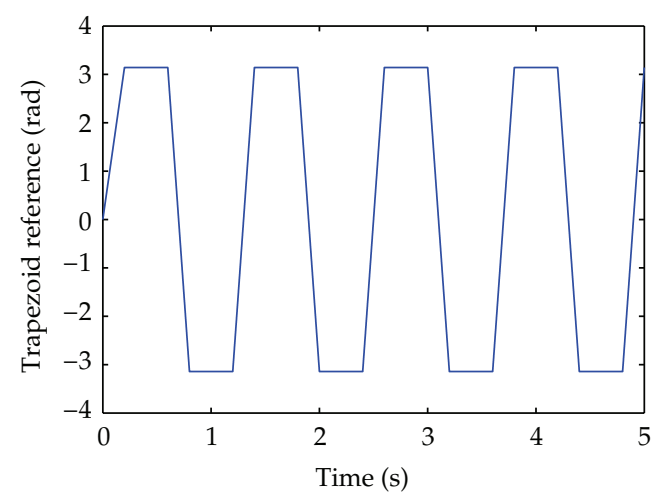

Figure 20: Trapezoid reference input.

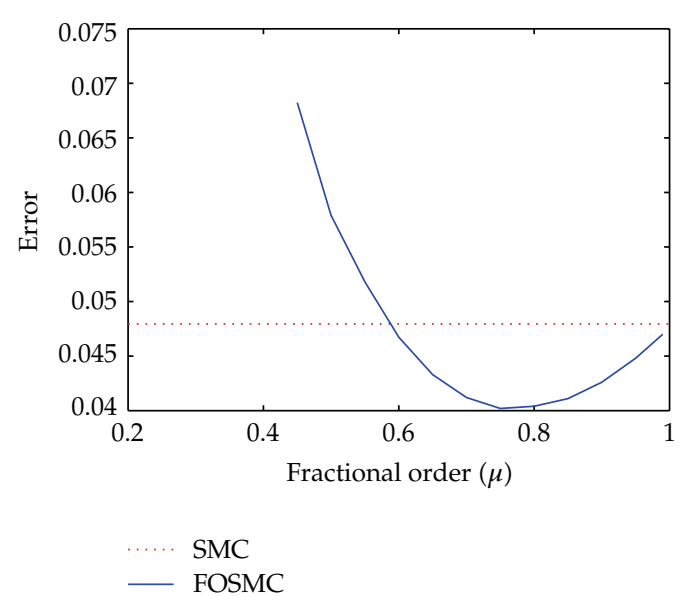

(a) Position error

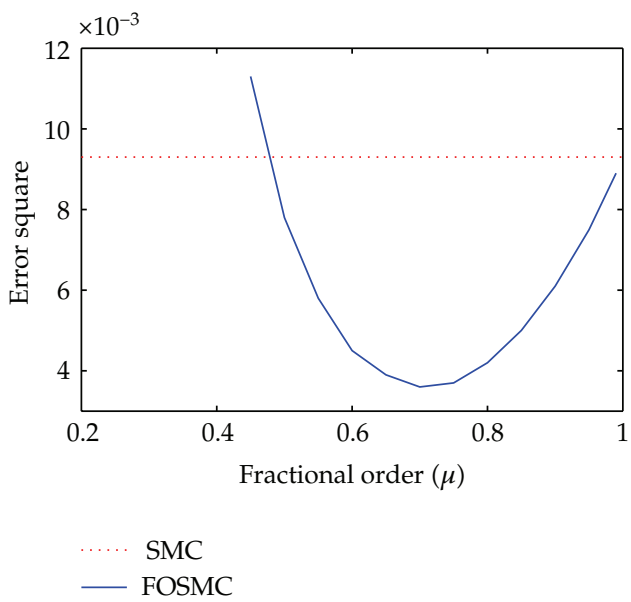

(b) Position error square

Figure 21: Position error and error square with sine reference input (2.3 Nm pulse disturbance, with saturation function).

Figure 16(a) shows the control output $i_{q}$ between $[0 \mathrm{~s}, 1.5 \mathrm{~s}]$, and the particular time period b, c, and d in Figure 16(a) is zoomed in as shown by Figures 16(b), 16(c), and 16(d), respectively. Figure 16(b) shows the control output $i_{q}$ between $[0 \mathrm{~s}, 0.02 \mathrm{~s}]$, during which the PMSM motor just started; Figure 16(c) shows the control output $i_{q}$ between [0.195 s, $0.22 \mathrm{~s}$ ] in this time period, the position output reaches the desired reference value; Figure 16(d) shows control output $i_{q}$ between $[0.60 \mathrm{~s}, 0.66 \mathrm{~s}]$, in which the external disturbance load is withdrawn. From Figures 16(b), 16(c), and 16(d), it can be seen that the control output $i_{q}$ of the proposed FOSMC method is more smooth than that of the conventional SMC method; in other words, the system chattering is eliminated to some degree by the FOSMC method.

\subsubsection{Simulation of Dynamic Position Response with Sinusoidal Input Signal}

In this simulation, the position reference is a sinusoidal trajectory with $\theta_{\text {ref }}(t)=\pi \sin (10 t)$ rad, the order of FOSMC is $\mu=0.6$, and the saturation function is used. A step disturbance load 
Table 1: PMSM specifications.

\begin{tabular}{lc}
\hline Features & Values \\
\hline Rated Voltage & $300 \mathrm{~V}$ \\
Maximum Speed $\left(\omega_{m}\right)$ & $2400 \mathrm{rpm}$ \\
Number of Poles $(P)$ & 4 \\
Phase Resistance $\left(R_{S}\right)$ & $2.46 \Omega$ \\
Winding Inductance $\left(L_{s}\right)$ & $4.233 \mathrm{mh}$ \\
Motor Inertia $(J)$ & $1.02 \times 10^{-3} \mathrm{Kg} \cdot \mathrm{m}^{2}$ \\
Friction Coefficient $(B)$ & $1.0 \times 10^{-4} \mathrm{~N} \cdot \mathrm{m} \cdot \mathrm{s} \cdot \mathrm{rad}^{-1}$ \\
Rotor Flux Linkages $\psi_{f}$ & $0.214 \mathrm{~Wb}$ \\
Torque $($ Te) & $5.25 \mathrm{Nm}$ \\
\hline
\end{tabular}

Table 2: Controller Performance.

\begin{tabular}{|c|c|c|}
\hline Controller Type & $\Delta e$ & $|\Delta e|^{2}$ \\
\hline SMC & 0.8766 & 0.2899 \\
\hline \multicolumn{3}{|l|}{ FOSMC } \\
\hline$\mu=0.35$ & 575.6 & 90180 \\
\hline$\mu=0.4$ & 351.9 & 34470 \\
\hline$\mu=0.45$ & 0.4186 & 0.0669 \\
\hline$\mu=0.5$ & 0.2695 & 0.0413 \\
\hline$\mu=0.55$ & 0.3014 & 0.0409 \\
\hline$\mu=0.6$ & 0.328 & 0.0463 \\
\hline$\mu=0.65$ & 0.363 & 0.0549 \\
\hline$\mu=0.7$ & 0.4057 & 0.0675 \\
\hline$\mu=0.75$ & 0.4575 & 0.085 \\
\hline$\mu=0.8$ & 0.517 & 0.1078 \\
\hline$\mu=0.85$ & 0.5895 & 0.1384 \\
\hline$\mu=0.9$ & 0.6738 & 0.1781 \\
\hline$\mu=0.95$ & 0.7716 & 0.2292 \\
\hline$\mu=0.99$ & 0.8492 & 0.2745 \\
\hline
\end{tabular}

of $3.1 \mathrm{Nm}$ is applied at $t=0.3 \mathrm{~s}$ and vanished at $t=0.35 \mathrm{~s}$, and another step disturbance load of $-3.1 \mathrm{Nm}$ is applied at $t=0.75 \mathrm{~s}$ and vanished at $t=0.8 \mathrm{~s}$. Figures $17(\mathrm{a})$ and $17(\mathrm{~b})$ show the position responses and position error, respectively. From the results, it is clear that the dynamic tracking error of the proposed FOSMC method is smaller than that of the conventional SMC method.

\subsubsection{Controller Performance with Different Fractional Orders}

We let the motor angle to track a sinusoidal trajectory $\theta_{\text {ref }}(t)=\pi \sin (10 t) \mathrm{rad}$, and a pulse load disturbance with $3.1 \mathrm{Nm}$ amplitude, $50 \%$ pulse width, and $100 \mathrm{~ms}$ period is applied to the PMSM the total running time is 5 seconds. Table 2 shows the controller performance of the conventional SMC method and the proposed FOSMC method with different fractional-order 


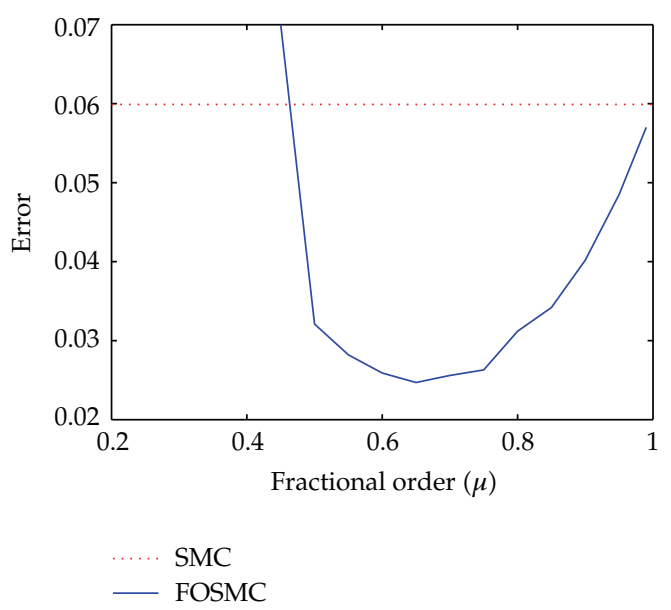

(a) Position error

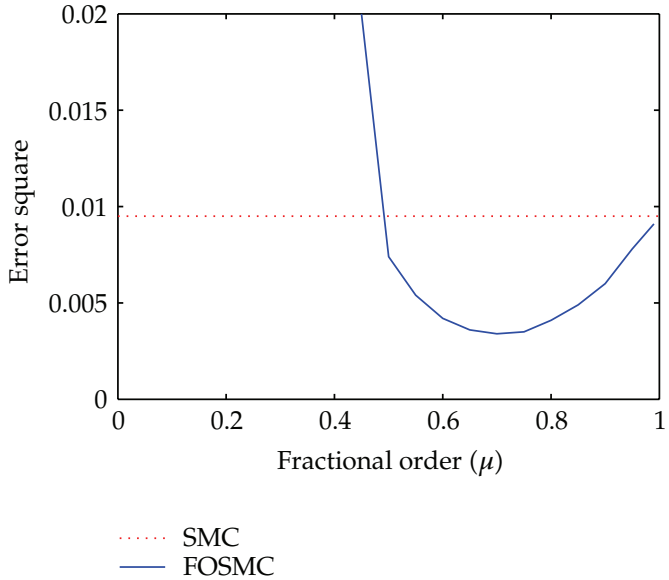

(b) Position error square

Figure 22: Position error and error square with sine reference input (2.3 Nm pulse disturbance, with sign function).

$\mu$, and the saturation function is adopted in both methods. In Table 2, the error $\Delta e$ and error square $|\Delta e|^{2}$ are defined as follows:

$$
\begin{aligned}
\Delta e & =\int\left|\theta_{\text {ref }}(t)-\theta_{m}(t)\right| d t, \\
|\Delta e|^{2} & =\int\left|\theta_{\text {ref }}(t)-\theta_{m}(t)\right|^{2} d t .
\end{aligned}
$$

From Table 2, it can be seen that when the fractional order of the proposed FOSMC method is set to small value, the performance is poor, but once the order is got value between $\mu \in$ $[0.45,0.95]$, then the position error and error square of the proposed FOSMC method are significantly smaller than those of conventional SMC method, especially when $\mu \in[0.5,0.6]$. This also means that the control performance of the FOSMC method can be improved by selecting a proper fractional-order $\mu$ and designing a corresponding fractional-order sliding surface.

\subsubsection{Controller Performance with Different Fractional-Order and Different Reference Input}

In this simulation, we check the effectiveness of the proposed FOSMC method to another position reference input and find the general regularity between the control performance and the different fractional-order $\mu$. Three position reference inputs, that is, sine wave, triangle wave, and trapezoid wave, are considered and, respectively, shown in Figures 18, 19, and 20. A pulse load disturbance with $50 \%$ pulse width, $100 \mathrm{~ms}$ period, and alternative amplitude of 3.1 Nm and $2.3 \mathrm{Nm}$ is applied to the PMSM. The total running time is 5 seconds.

In the simulation, for each position reference input, the position error and error square of the proposed FOSMC method and the convention SMC method are regarded as the control 


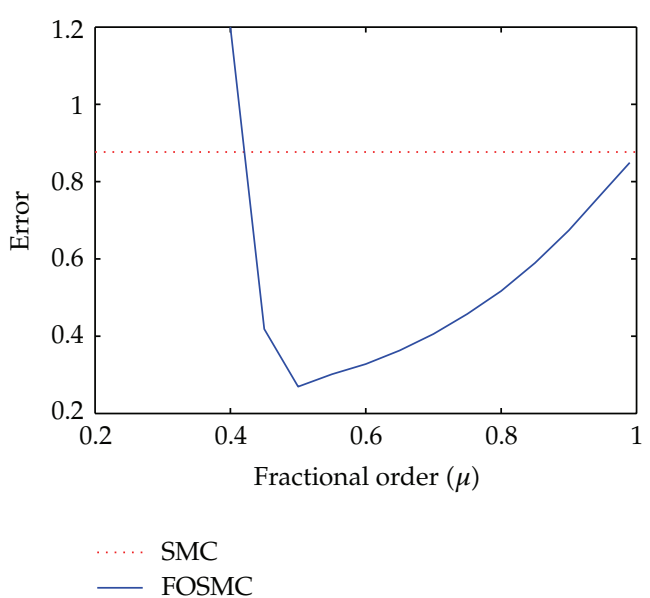

(a) Position error

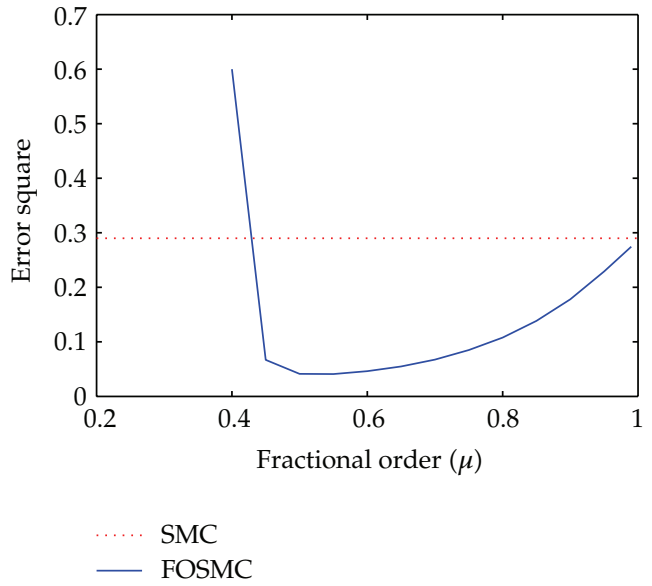

(b) Position error square

Figure 23: Position error and error square with sine reference input ( $3.1 \mathrm{Nm}$ pulse disturbance, with saturation function).

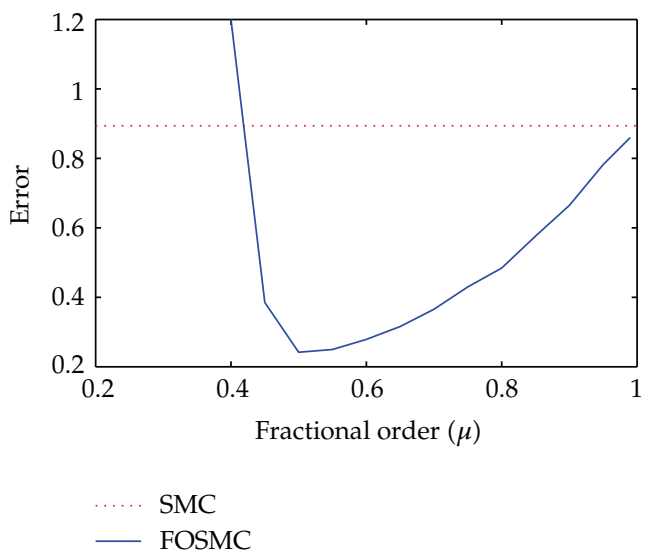

(a) Position error

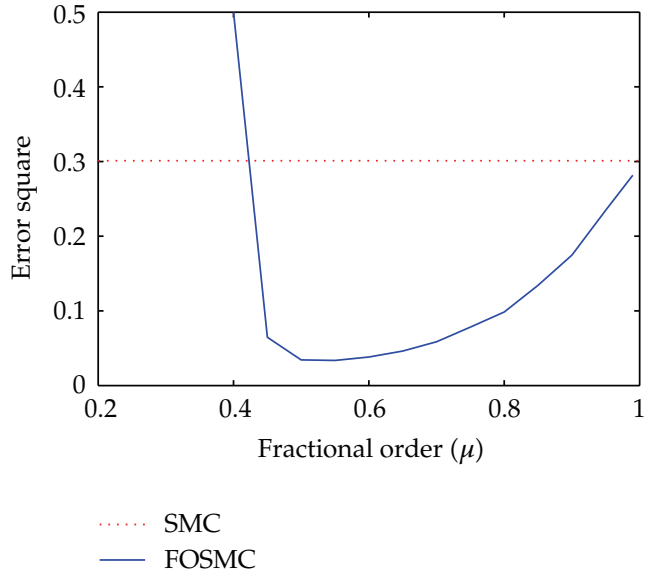

(b) Position error square

Figure 24: Position error and error square with sine reference input (3.1 Nm pulse disturbance, with sign function).

performance. The amplitude of the pulse load disturbance is set as $2.3 \mathrm{Nm}$ and $3.1 \mathrm{Nm}$, respectively, which means that the system is stable under the load disturbance of $2.3 \mathrm{Nm}$ and unstable under the load disturbance of $3.1 \mathrm{Nm}$. Moreover, under the two kinds of load amplitude, the saturation function and sign function are considered, respectively.

For comparison convenience, in Figures 21, 22, 23, 24, 25, 26, 27, 28, 29, 30, 31, and 32 , the red dot line represents the error or error square obtained by the conventional SMC method, and it has no relationship with the fractional-order $\mu$, while the green solid line is the error and error square got by the proposed FOSMC method with different fractional-order $\mu$. 


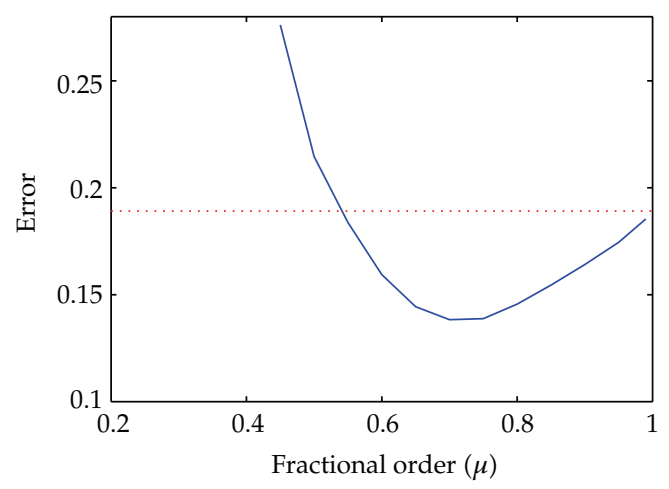

$\mathrm{SMC}$ - FOSMC

(a) Position error

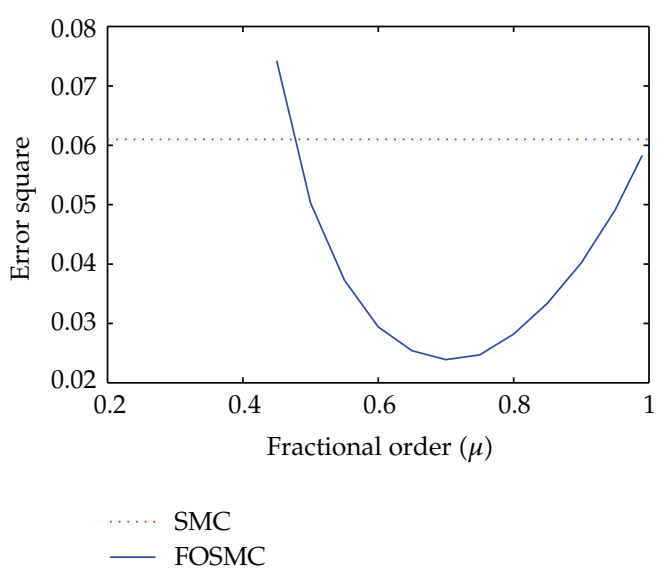

(b) Position error square

Figure 25: Position error and error square with triangle reference input (2.3 Nm pulse disturbance, with saturation function).

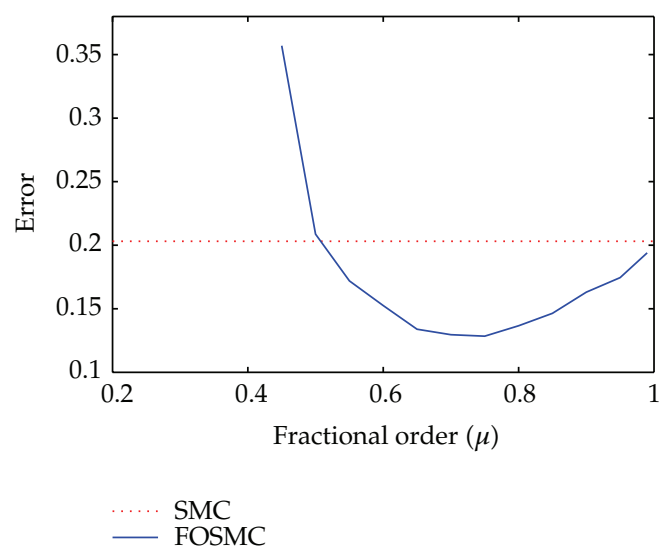

(a) Position error

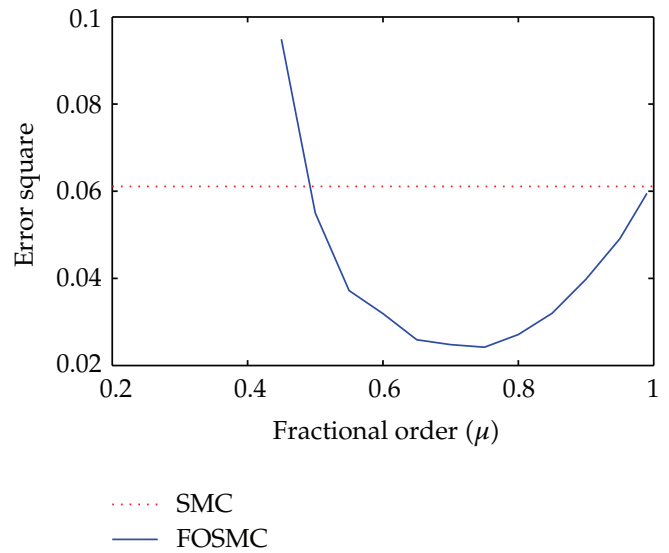

(b) Position error square

Figure 26: Position error and error square with triangle reference input $(2.3 \mathrm{Nm}$ pulse disturbance, with sign function).

From Figures 21-32, it is clear that when $\mu \in\left(0, \mu^{*}\right)$, then the error or error square of the FOSMC method is bigger than that of the conventional SMC method, but when $\mu \in$ $\left[\mu^{*}, 1\right)$, the error or error square of the FOSMC method is significantly smaller than that of the conventional SMC method. Moreover, from Figures 21-32, one can see that the value of $\mu^{*}$ is around 0.5 ; this also means that when the fractional-order $\mu \in[0.5,1)$, then the proposed FOSMC method outperforms the conventional SMC method.

In fact, from the simulation results shown from Figures 21-32, it can be seen that the best selection range for $\mu$ is $[0.5,0.6]$. 


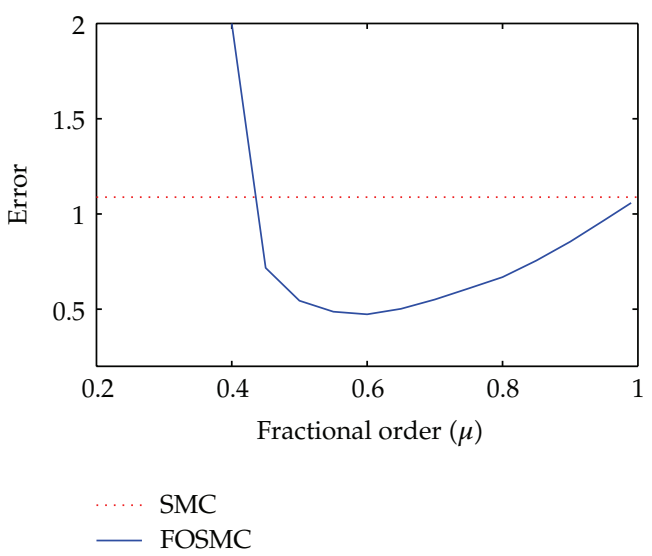

(a) Position error

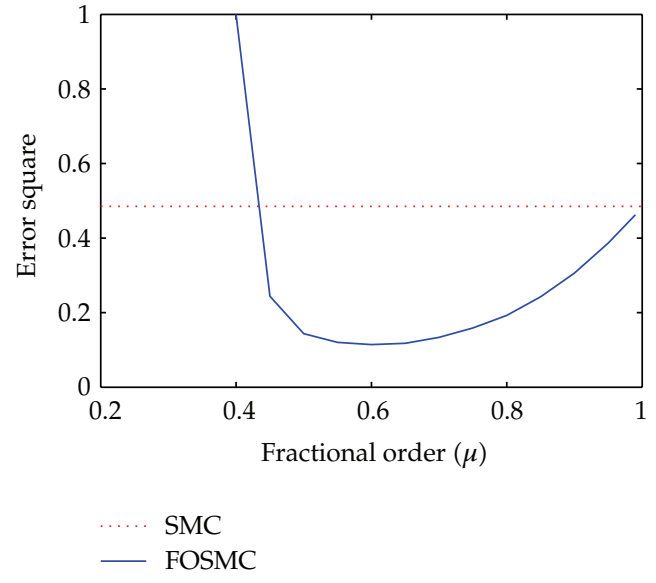

(b) Position error square

Figure 27: Position error and error square with triangle reference input (3.1 Nm pulse disturbance, with saturation function).

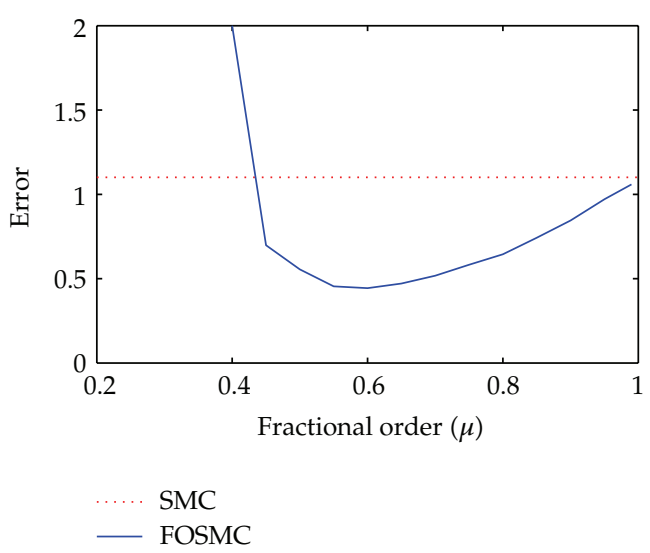

(a) Position error

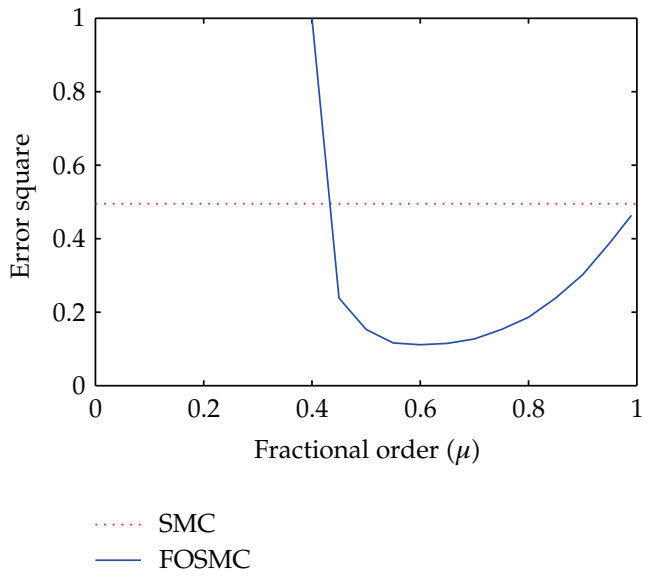

(b) Position error square

Figure 28: Position error and error square with triangle reference input (3.1 Nm pulse disturbance, with sign function).

\section{Guidance for Parameters Selection and Design of FOSMC}

In the proposed FOSMC method, there are five parameters, that is, $k_{p}, k_{d}, k, \varepsilon$, and $\mu$, which need to be designed. From the above analyses and numerical simulation results, one can select and design the five parameters through the following procedures:

(i) select a value in the range $[0.5,0.6]$ or $[0.5,1)$ for the fractional-order $\mu$;

(ii) estimate the maximum load disturbance $T_{L-\max }$;

(iii) according to the $T_{L-\max }$ and (5.21), compute the value range of parameter $k$ and then select a suitable value for $k$; 


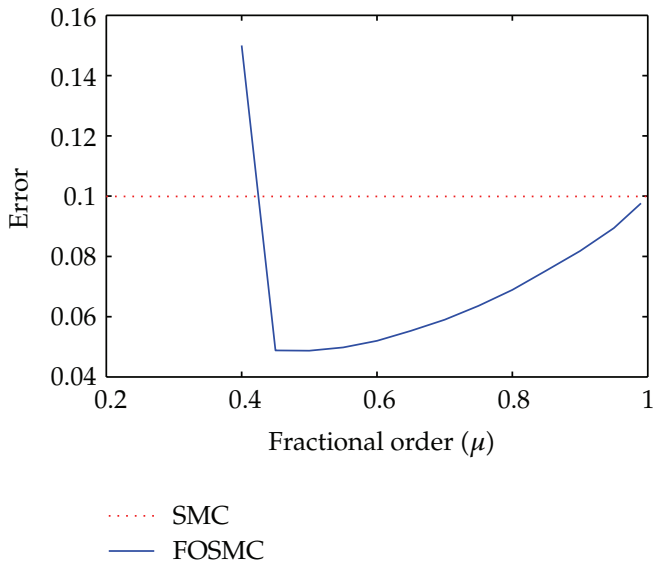

(a) Position error

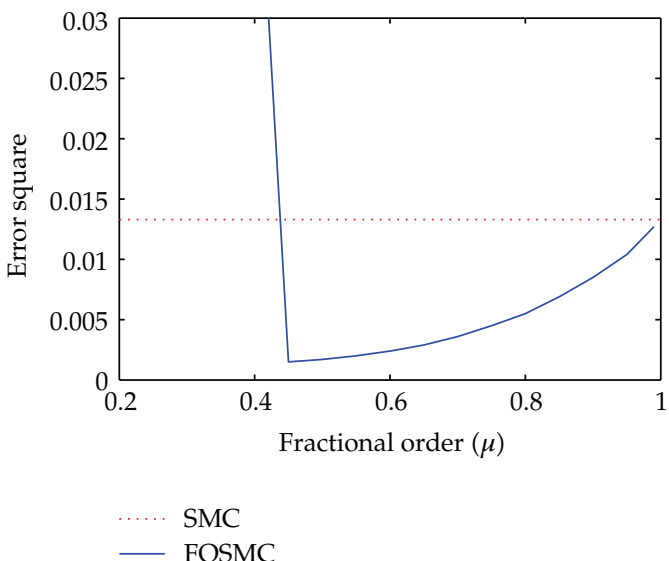

(b) Position error square

Figure 29: Position error and error square with trapezoid reference input ( $2.3 \mathrm{Nm}$ pulse disturbance, with saturation function).

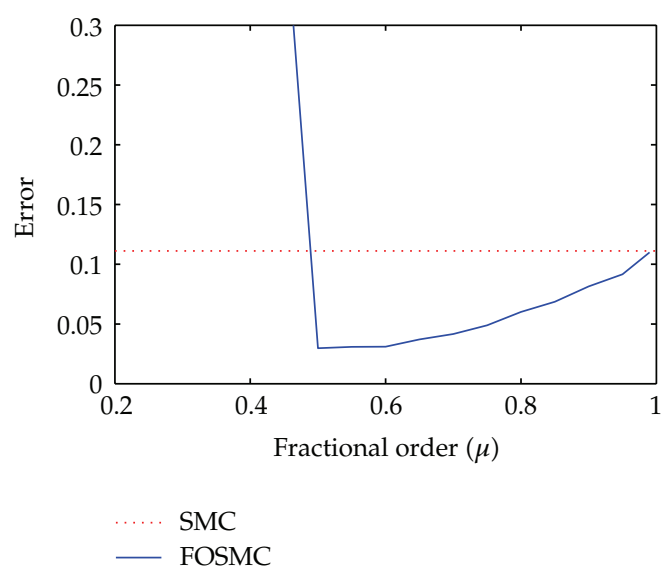

(a) Position error

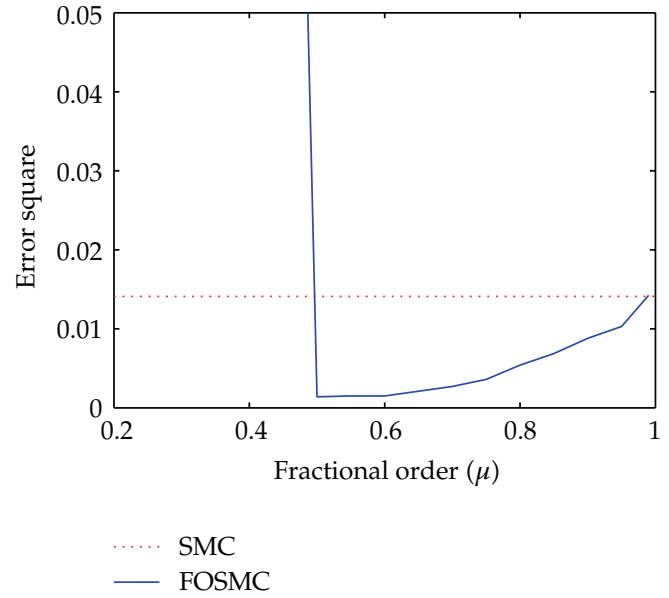

(b) Position error square

Figure 30: Position error and error square with trapezoid reference input ( $2.3 \mathrm{Nm}$ pulse disturbance, with sign function).

(iv) because $k_{d}$ is the coefficient of fractional-order differentiation of position error $x_{1}$, if the value of $k_{d}$ is big, it will be too sensitive to the variation of position error $x_{1}$ and cause oscillation. In general, $k_{d}$ can be set as a suitable small value, for example, in this paper $k_{d}=1$;

(v) with the maximum permissible position error $x_{1}$ of the PMSM system and the parameter $k$ designed by the procedure (iii), then parameters $\varepsilon$ and $k_{p}$ can be designed and selected according to (5.28). 


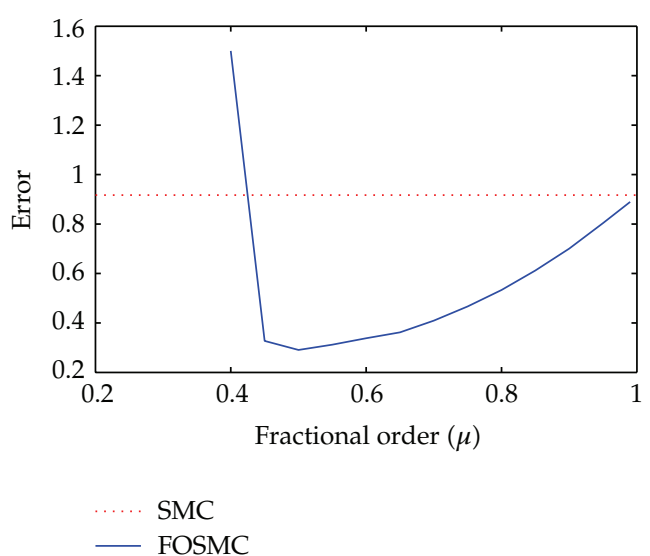

(a) Position error

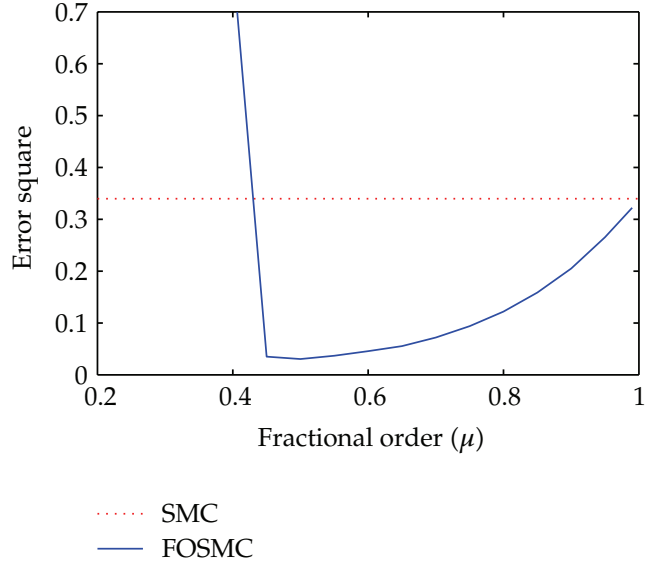

(b) Position error square

Figure 31: Position error and error square with trapezoid reference input (3.1 Nm pulse disturbance, with saturation function).

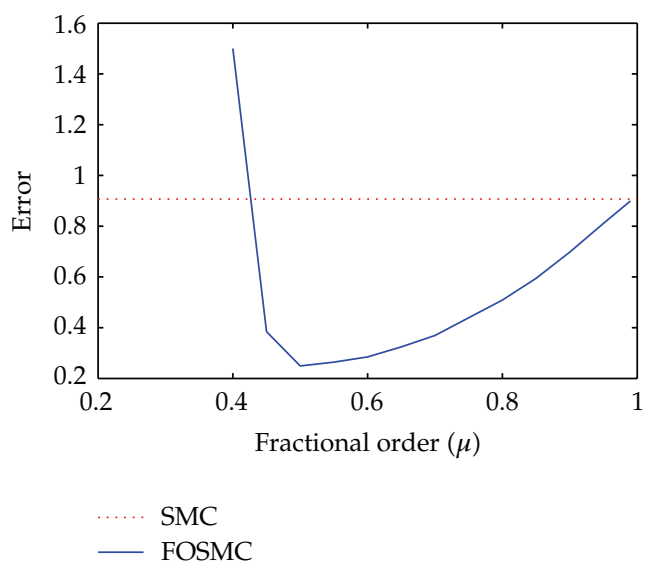

(a) Position error

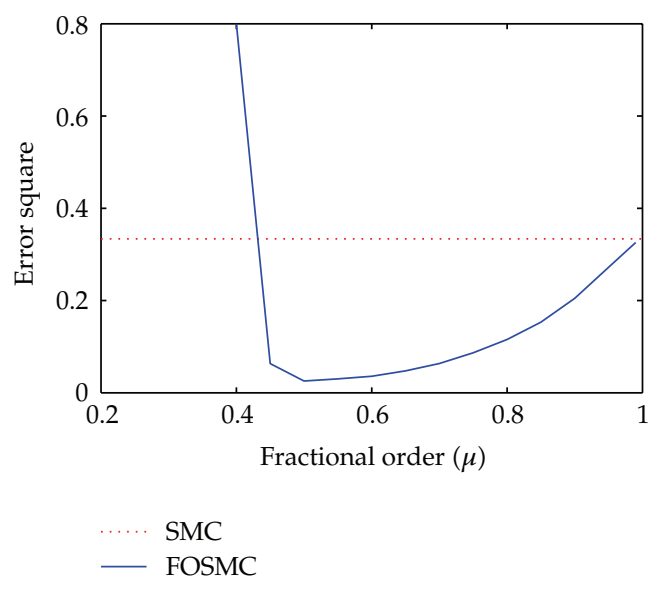

(b) Position error square

Figure 32: Position error and error square with trapezoid reference input (3.1 Nm pulse disturbance, with sign function).

\section{Conclusions}

A new and systematic design of the fractional-order sliding mode controller (FOSMC) for PMSM position control system is presented. By selecting a proper fractional-order $\mu$ and designing a fractional-order sliding surface, the control performance such as control precision and system robustness of the proposed FOSMC method is distinctly more excellent than that of the conventional SMC method, because an extra fractional order, the real parameters $\mu$, is involved. The robustness of the proposed FOSMC method is analyzed in detail, and the guidance for parameters selection and design is given. The numerical simulation results demonstrate the effectiveness and robustness of the proposed FOSMC method. 


\section{Acknowledgments}

This work is supported by the National Natural Science Foundation of China (61104085), the Educational Committee Foundation of Jiangsu Province (11KJB510005), and the Jiangsu Province Six Talents Project JXQC-139(103).

\section{References}

[1] K. K. Shyu, C. K. Lai, Y. W. Tsai, and D. I. Yang, “A newly robust controller design for the position control of permanent-magnet synchronous motor," IEEE Transactions on Industrial Electronics, vol. 49, no. 3, pp. 558-565, 2002.

[2] F. J. Lin, "Real-time IP position controller design with torque feedforward control for PM synchronous motor," IEEE Transactions on Industrial Electronics, vol. 44, no. 3, pp. 398-407, 1997.

[3] F. J. Lin and Y. S. Lin, "A robust PM synchronous motor drive with adaptive uncertainty observer," IEEE Transactions on Energy Conversion, vol. 14, no. 4, pp. 989-995, 1999.

[4] C. K. Lai and K. K. Shyu, "A novel motor drive design for incremental motion system via slidingmode control method," IEEE Transactions on Industrial Electronics, vol. 52, no. 2, pp. 499-507, 2005.

[5] R. Errouissi and M. Ouhrouche, "Nonlinear predictive controller for a permanent magnet synchronous motor drive," Mathematics and Computers in Simulation, vol. 81, no. 2, pp. 394-406, 2010.

[6] A. M. Harb, "Nonlinear chaos control in a permanent magnet reluctance machine," Chaos, Solitons $\mathcal{E}$ Fractals, vol. 19, no. 5, pp. 1217-1224, 2004.

[7] S. Zhao and K. K. Tan, "Adaptive feedforward compensation of force ripples in linear motors," Control Engineering Practice, vol. 13, no. 9, pp. 1081-1092, 2005.

[8] J. Zhou and Y. Wang, "Real-time nonlinear adaptive backstepping speed control for a PM synchronous motor," Control Engineering Practice, vol. 13, no. 10, pp. 1259-1269, 2005.

[9] T. L. Hsien, Y. Y. Sun, and M. C. Tsai, " $H^{\infty}$ control for a sensor less permanent magnet synchronous drive," IEE Proceedings_Electric Power Applications, vol. 144, no. 3, pp. 173-181, 1997.

[10] A. R. Ghafari-Kashani, J. Faiz, and M. J. Yazdanpanah, "Integration of non-linear $H^{\infty}$ and sliding mode control techniques for motion control of a permanent magnet synchronous motor," IET Electric Power Applications, vol. 4, no. 4, pp. 267-280, 2010.

[11] T. S. Lee, C. H. Lin, and F. J. Lin, "An adaptive $H_{\infty}$ controller design for permanent magnet synchronous motor drives," Control Engineering Practice, vol. 13, no. 4, pp. 425-439, 2005.

[12] A. Mezouar, M. K. Fellah, and S. Hadjeri, "Adaptive sliding mode observer for induction motor using two-time-scale approach," Electric Power Systems Research, vol. 77, no. 5-6, pp. 604-618, 2007.

[13] N. Inanc and V. Ozbulur, "Torque ripple minimization of a switched reluctance motor by using continuous sliding mode control technique," Electric Power Systems Research, vol. 66, no. 3, pp. 241$251,2003$.

[14] C. F. J. Kuo, C. H. Hsu, and C. C. Tsai, "Control of a permanent magnet synchronous motor with a fuzzy sliding-mode controller," The International Journal of Advanced Manufacturing Technology, vol. 32, no. 7-8, pp. 757-763, 2007.

[15] C. Elmas and O. Ustun, "A hybrid controller for the speed control of a permanent magnet synchronous motor drive," Control Engineering Practice, vol. 16, no. 3, pp. 260-270, 2008.

[16] F. F. M. El-Sousy, "Robust wavelet-neural-network sliding-mode control system for permanent magnet synchronous motor drive," IET Electric Power Applications, vol. 5, no. 1, pp. 113-132, 2011.

[17] Y. Feng, J. F. Zheng, X. H. Yu, and N. V. Truong, "Hybrid terminal sliding-mode observer design method for a permanent-magnet synchronous motor control system," IEEE Transactions on Industrial Electronics, vol. 56, no. 9, pp. 3424-3431, 2009.

[18] S. H. Chang, Y. H. Ting, P. Y. Chen, and S. W. Hung, "Robust current control-based sliding mode control with simple uncertainties estimation in permanent magnet synchronous motor drive systems," IET Electric Power Applications, vol. 4, no. 6, pp. 441-450, 2010.

[19] I. Podlubny, "Fractional-order systems and $P I^{\lambda} D^{\mu}$-controllers," IEEE Transactions on Automatic Control, vol. 44, no. 1, pp. 208-214, 1999.

[20] Y. Q. Chen, I. Petras, and D. Y. Xue, "Fractional order control-a tutorial," in Proceedings of the American Control Conference (ACC'09), pp. 1397-1411, June 2009. 
[21] A. Oustaloup, F. Levron, B. Mathieu, and F. M. Nanot, "Frequency-band complex noninteger differentiator: characterization and synthesis," IEEE Transactions on Circuits and Systems I, vol. 47, no. 1, pp. 25-39, 2000.

[22] K. B. Oldham and J. Spanier, The Fractional Calculus, Academic Press, New York, NY, USA, 1974.

[23] I. Podlubny, Fractional Differential Equations, Academic Press, San Diego, Calif, USA, 1999.

[24] Y. Q. Chen, H. S. Ahn, and I. Podlubny, “Robust stability check of fractional order linear time invariant systems with interval uncertainties," Signal Processing, vol. 86, no. 10, pp. 2611-2618, 2006.

[25] M. Ö. Efe, "Fractional fuzzy adaptive sliding-mode control of a 2-DOF direct-drive robot arm," IEEE Transactions on Systems, Man, and Cybernetics B, vol. 38, no. 6, pp. 1561-1570, 2008.

[26] H. Delavari, A. N. Ranjbar, R. Ghaderi, and S. Momani, "Fractional order control of a coupled tank," Nonlinear Dynamics, vol. 61, no. 3, pp. 383-397, 2010.

[27] H. S. Li, Y. Luo, and Y. Q. Chen, "A fractional order proportional and derivative (FOPD) motion controller: tuning rule and experiments," IEEE Transactions on Control Systems Technology, vol. 18, no. 2, pp. 516-520, 2010.

[28] Y. Li, Y. Q. Chen, and I. Podlubny, "Stability of fractional-order nonlinear dynamic systems: lyapunov direct method and generalized Mittag-Leffler stability," Computers \& Mathematics with Applications, vol. 59, no. 5, pp. 1810-1821, 2010.

[29] D. Baleanu, H. Mohammadi, and S. Rezapour, "Positive solutions of an initial value problem for nonlinear fractional differential equations," Abstract and Applied Analysis, vol. 2012, Article ID 837437, 7 pages, 2012.

[30] D. Baleanu, K. Diethelm, E. Scalas, and J. J. Trujillo, Fractional Calculus Models and Numerical Methods, World Scientific, New York, NY, USA, 2012.

[31] D. Bleanu, O. G. Mustafa, and R. P. Agarwal, "Asymptotically linear solutions for some linear fractional differential equations," Abstract and Applied Analysis, vol. 2010, Article ID 865139, 8 pages, 2010.

[32] F. Jarad, T. Abdeljawad, and D. Baleanu, "On Riesz-Caputo formulation for sequential fractional variational principles," Abstract and Applied Analysis, vol. 2012, Article ID 890396, 15 pages, 2012.

[33] M. O. Efe, "A fractional order adaptation law for integer order sliding mode control of a 2DOF robot," in Proceedings of the International Workshops on New Trends in Science and Technology (NTST 08) and the Workshop Fractional Differentiation and Its Applications (FDA 09), Ankara, Turkey, November 2008.

[34] S. H. Hosseinnia, R. Ghaderi, N. A. Ranjbar, M. Mahmoudian, and S. Momani, "Sliding mode synchronization of an uncertain fractional order chaotic system," Computers $\mathcal{E}$ Mathematics with Applications, vol. 59, no. 5, pp. 1637-1643, 2010.

[35] B. T. Zhang and Y. Pi, "Robust fractional order proportion-plus-differential controller based on fuzzy inference for permanent magnet synchronous motor," IET Control Theory \& Applications, vol. 6, no. 6, pp. 829-837, 2012.

[36] Y. Luo, Y. Q. Chen, H. S. Ahn, and Y. Pi, "Fractional order robust control for cogging effect compensation in PMSM position servo systems: stability analysis and experiments," Control Engineering Practice, vol. 18, no. 9, pp. 1022-1036, 2010.

[37] Y. Q. Chen, “Impulse response invariant discretization of fractional order integrators/differentiators is to compute a discrete-time finite dimensional $(\mathrm{z})$ transfer function to approximate $\mathrm{s}^{r}$ with $\mathrm{r}$ a real number," Category: Filter Design and Analysis, MATLAB Central, 2008, http://www.mathworks .com/matlabcentral/ fileexchange/loadFile.do objectId=21342 objectType=FILE. 


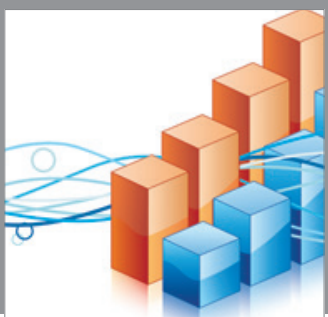

Advances in

Operations Research

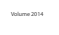

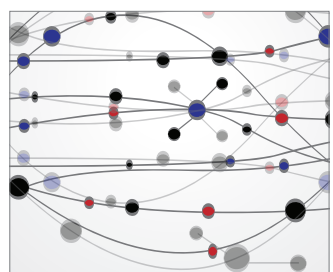

\section{The Scientific} World Journal
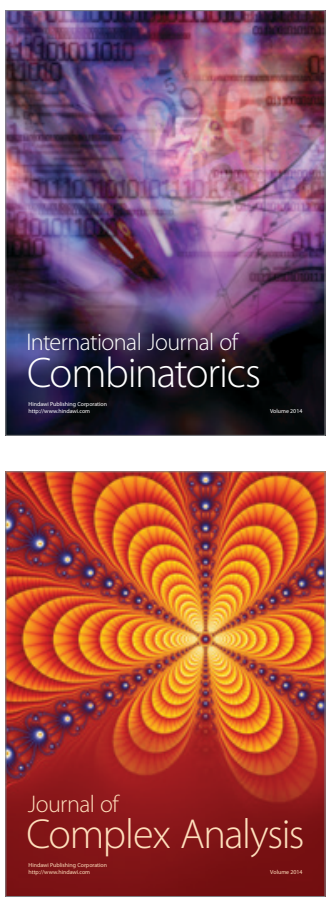

International Journal of

Mathematics and

Mathematical

Sciences
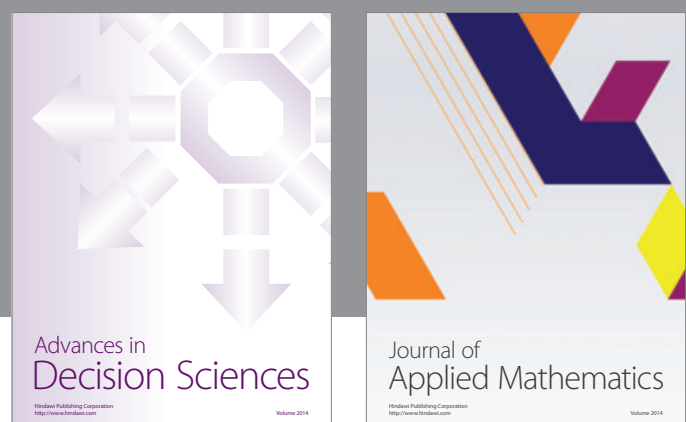

Journal of

Applied Mathematics
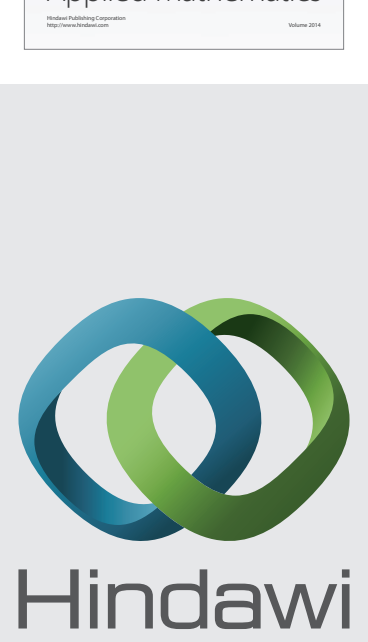

Submit your manuscripts at http://www.hindawi.com
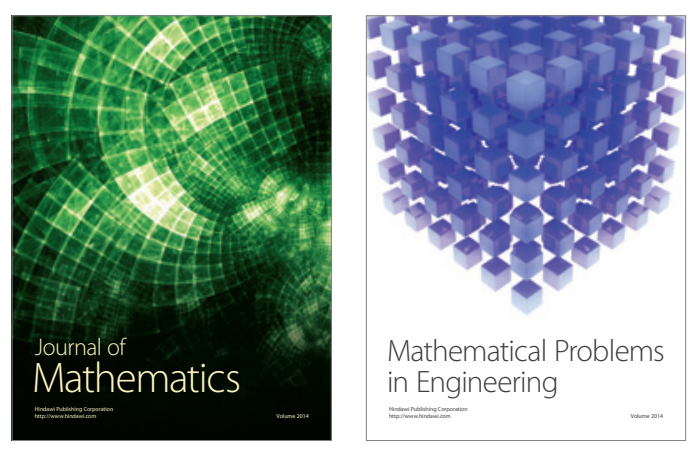

Mathematical Problems in Engineering
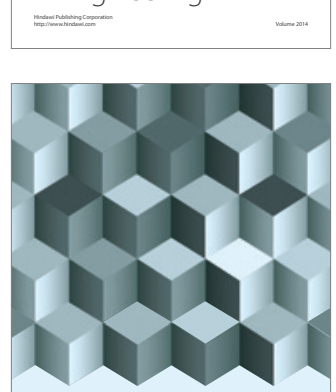

Journal of

Function Spaces
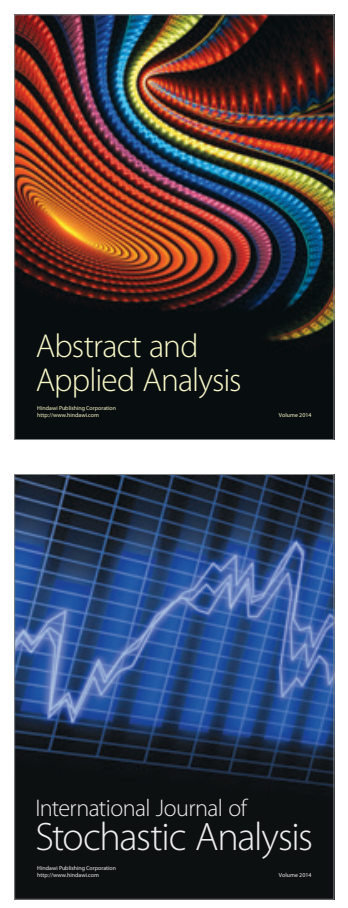

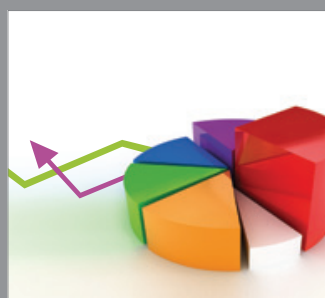

ournal of

Probability and Statistics

Promensencen
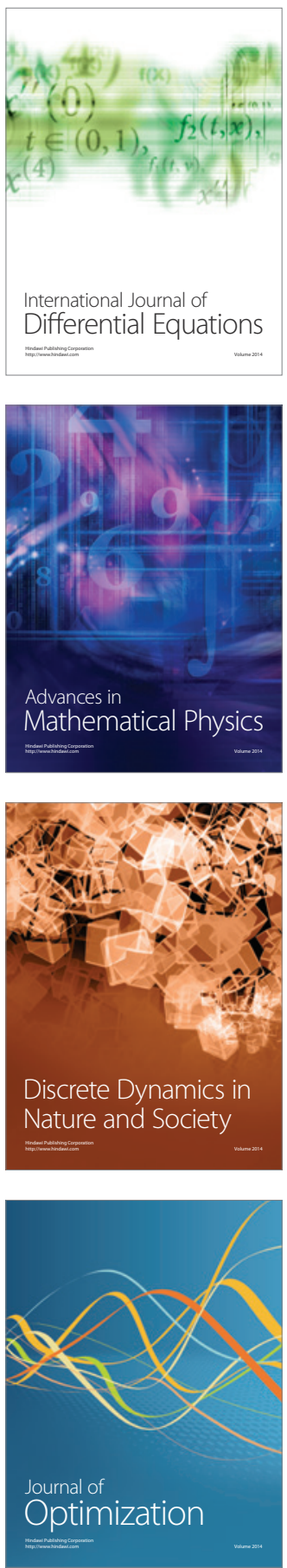